\title{
Code sacrificiel et catégories de pensée chez les
} Bobo de Haute-Volta

Sacrificial code and categories of thought among the Bobo of Upper Volta

\section{Guy Le Moal}

\section{(2) OpenEdition}

\section{Journals}

Édition électronique

URL : http://journals.openedition.org/span/524

DOI : 10.4000/span.524

ISSN : 2268-1558

\section{Éditeur}

École pratique des hautes études. Sciences humaines

\section{Édition imprimée}

Date de publication : 1 avril 1984

Pagination : 9-64

ISSN : 0294-7080

\section{Référence électronique}

Guy Le Moal, « Code sacrificiel et catégories de pensée chez les Bobo de Haute-Volta », Systèmes de pensée en Afrique noire [En ligne], 6 | 1984, mis en ligne le 04 juin 2013, consulté le 30 avril 2019. URL http://journals.openedition.org/span/524; DOI : 10.4000/span.524 


\author{
$\begin{array}{llllllllllllll}C & O & D & E & S & A & C & R & I & F & I & C & I & E\end{array}$ \\ E T C A TEGORIES DE PEN SE E

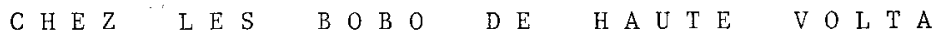 \\ par
}

Guy Le Moa1

Dans un précédent article ${ }^{1}$, nous avons traité des rites sacrificiels tels qu'ils se présentent, très concrètement, dans la pratique constante du culte chez les Bobo. Passant en revue les phases successives du procès sacrificiel -- dépôt de la cendre, discours, 1ibations, immolation des victimes, nouvelles offrandes et enfin consommation -nous avons pris soin d'énumérer et de décrire les espèces animales et les matières végétales réservées aux fins rituelles. Nous avons alors constaté que les matières et espèces manipulées dans le cadre du sacrifice étaient l'objet de choix bien précis fondés sur la sélection de caractères donnés (sexe, couleur...). Nous avons vu également s'esquisser des classifications et même des hiérarchies tandis qu'un jeu complexe de relations pouvait se déceler entre les divers éléments choisis et, ce qui est encore plus intéressant, entre ces derniers et les entités spirituelles destinataires.

Puisqu'ainsi s'affirmait l'existence d'un véritable code, c'est à dire d'un système organisé de conventions, il s'imposait d'en tenter le déchiffrement et, au-delà, d'en analyser les éléments constitutifs aux fins d'en élucider le sens.

1. Introduction à une étude du sacrifice chez les Bobo de Haute Volta, Systèmes de pensée en Afrique noire, Cahier 5, Le sacrifice IV, 1981, pp. 99-125. Nous renverrons à cet article sous la forme suivante : "GLM 1981 : (page)". 


\section{LES FAITS}

C'est à l'échelle du village, catégorie sociale pertinente chez les Bobo -- nous l'avons déjà souligné (GLM 1981) --, que notre recherche s'est accomplie.

Nous disposions, pour le village choisi (Kurumani), d'un recensement exhaustif des"lieux sacrés". Il était le résultat d'investigations systématiquement menées par nous pour tenter de circonscrire 1 'ensemble du champ des manifestations religieuses tant aux niveaux lignager et clanique qu'au niveau communautaire (foroba). villageois.

Chacun des lieux sacrés existants avait été localisé et inscrit sur des cartes du village, leur aspect matériel (typologie des autels notamment) étant dûment indiqué. L'ensemble de ces documents -- 1istes et cartes - a été publié dans notre ouvrage sur la religion bobo ${ }^{2}$, il y figure à titre de "pièces à conviction" et vient à 1'appui des faits relatés dans le texte.

Issu du même ouvrage, nous disposions également d'un "organigramme" des cultes qui nous offrait, au terme d'un panorama des mythes et des divinités, un cadre de classement des principales entités spirituelles bobo (GLM 1980 : fig. 6, p. 159).

Ayant pris par ailleurs le soin d'indentifier exactement quelle entité concernait le culte qui se déroulait sur chacun des lieux sacrés recensés, il nous était facile de mettre en rapport les deux documents en question et d'établir ainsi une liste unique des lieux sacrés et autels classés en fonction des entités y demeurant.

C'est cette liste qui a servi de base à notre enquête. Un certain traitement des données s'est cependant imposé au préalable afin d'adapter au projet spécifique visé -- étude des modalités du sacrifice -- ce qui avait été collecté dans le cadre d'une recherche

2. Le Moal G., Les Bobo. Nature et fonction des masques, Paris, Travaux et documents de $1^{\prime}$ ORSTOM, $\mathrm{n}^{\circ} 121,1980, \mathrm{X}+535 \mathrm{p} ., 37 \mathrm{fig} ., 36$ photos, 3 cartes H.T., ind., biblio.

Voir hors texte : fig. 36 et tableau 11 : carte et 1 iste des lieux sacrés intra muros; fig. 37 et tableau 13 : carte et liste des lieux sacrés extro muros. D'une façon générale, pour plus de précisions sur ces lieux et sur la nature des entités spirituelles citées dans le présent article, on pourra se reporter à cet ouvrage. Occasionnellement, nous y renverrons cependant ici sous la forme suivante : "GLM 1980 : (page)". 
beaucoup plus globale. Ensuite, par touches successives, quelques modifications ont été également introduites, dans le cours même du travail, au fur et à mesure que les informations recueillies apportaient des éclairages nouveaux, des précisions, voire des points de vue différents tant sur la personnalité de certaines entités que sur leur rang.

C'est dans sa forme définitive que nous proposons ici la liste des autels et des entités (tableau 1). Dans l'ensemble, les remaniements opérés, loin d'altérer les lignes du tableau général des croyances, telles que nous les avions dégagées dans notre ouvrage sur les Bobo, ont eu tendance au contraire à en accuser le tracé et c'est avec une vigueur accrue que transparaissent, au bout du compte, les principes moteurs du système de pensée bobo. D'emblée, s'est ainsi affirmé le fait que le sacrifice était un lieu privilégié d'expression du dogme et que son étude offrait la meilleure des voies pour accéder à son intelligence.

1.1. Du premier de nos deux documents de base (1iste des "lieux sacrés", GLM 1980 : tab. 11 et 13), il nous a fallu tout d'abord éliminer une série de lieux qui n'étaient pas ordinairement destinataires des sacrifices. Ces lieux sont en relation avec la pratique du culte d'une entité $^{3}$ et ils se trouvent investis d'une certaine sacralité par une sorte d'effet de contiguīté ; ils ne peuvent être cependant considérés pleinement comme des entités spirituelles et ne font d'ailleurs pas l'objet d'un culte propre.

3. Nombre d'entre eux sont placés sous le signe du culte de dwo. Ce sont des lieux en rapport avec les rites initiatiques : places où ils se déroulent ( $t a b .13: 7,32,33,52,13,31$ ); places des danses (tab. $13: 17,18-$ tab. $11: 13$ ); lieux de réunion de classes d'âge (tab. $11: 6,16,45-$ tab. $13: 8,15,45$ ). Ce sont aussi des 1 ieux en rapport avec les masques eux-mêmes : lieux où ils sont fabriqués ( tab. 13 : $34,39,40,64,65,67,68,69,24)$; lieux où 1 'on change de porteur (tab. $13: 38$ ) ; lieux où $1^{\prime}$ on dépose (tab. $11: 22$, 40) et où 1 'on détruit les masques ( $t a b .13: 63,65)$.

Il y a également des lieux en rapport avec d'autres cultes que celui de $d w o$ : cultes tribaux (tab. 13:62), de sindo (tab. $11: 20$ ), des kôndigö (tab. 11 : 34). Enfin ont été supprimés de 1a liste quelques objets (tab. 11:3,4,7) et quelques arbres plus ou moins sacralisés (tab. $13: 14,25,30)$. 
Neuf cultes d'entités très secondaires ont également dû être retirés de notre liste : trois d'entre eux parce qu'ils sont de ressort purement privé (zakane) et assez mal connus ${ }^{4}$; trois autres parce qu'ils sont d'origine étrangère ${ }^{5}$; les trois derniers parce qu'ils sont abandonnés depuis plusieurs années ${ }^{6}$.

C'est en définitive sur le culte de 61 entités parfaitement spécifiques et sur les sacrifice qu'elles reçoivent qu'a porté notre recherche.

Une fois établie cette 1 iste, il est apparu très vite la nécessité d'introduire un ordre dans 1'énumération des entités et cela pour répondre à une exigence taxinomique continuellement exprimée par le discours théogonique bobc tout autant que pour satisfaire à la règle scientifique. Nous avions, certes, élaboré déjà un cadre général de classement des entités au cours đe nos précédents travaux (cf. supra), cependant, en même temps que le recueil des données relatives aux actes sacrificiels confirmait l'exactitude de ce classement, certains manques apparaissaient dans l'expression surtout du jeu des relations et influences réciproques. Ce n'est pas 1 'un des moindres mérites de cette enquête sur le sacrifice que de nous avoir montré l'existence d'une véritable systématique et donné les moyens d'échafauder une typologie des entités spirituelles.

De cette typologie, nous retiendrons l'essentiel.

II existe :

a) Des entités spirituelles pleinement individualisécs. Elles sont identifiées par un nom personnel et par un autel voué à leur culte exclusif.

Parmi ces entités, quelques-unes ont une importance particulière parce qu'elles se présentent comme les "figures initiales"7 de

4. Kwere, sî nono, batra (tab. $13: 26,54,60$ ).

5. bambara (tab. $11: 31,32-35$ ) et culte syncrétique originaire de San (tab. $13: 3$ ).

6. kola zo (tab. $13: 50$ ) et deux cultes d'enfants : kalsisi et dodoroma (tab. $11: 28,38)$.

7. Le concept de "figure" et la distinction qui existe entre la "figure initiale"d'une entité et ses "figures ultérieures" est un des éléments originaux de la réflexion religieuse bobo. La figure initiale s'identifie avec la manifestation première de l'entité; cette révélation 
ce qu'on peut considérer comme étant des véritables catégories de la pensée bobo. Chacune de ces figures a pris en effet valeur de paradigme et elle est éponyme de l'une des 8 catégories suivantes :

$\begin{array}{ll}1 \text { wuro } & \text { la divinité suprême, le créateur } \\ 2 \text { kwere } & \text { la foudre, 1'arme de Dieu } \\ 3 \text { zo } & \text { l'élément aquatique, les eaux courantes } \\ 4 \text { soxo } & \text { la brousse, le végétal à 1'état sauvage } \\ 5 \text { dwo } & \text { le mainteneur de 1'ordre divin, le masque } \\ 6 \text { funanyone } 8 & \text { les "esprits" immatériels } \\ 7 \text { wiyaxe } & \text { les "génies", êtres corporels } \\ 8 \text { bqrè } & \text { les ancêtres }\end{array}$

A la suite de ces entités primordiales, placées en tête de liste de chacune des catégories, viennent ce que nous appelons les diverses "figures ultérieures" de ces mêmes entités. Le plus souvent, il s'agit des manifestations "réitérées" d'une entité, ainsi que des cultes nouveaux (et toujours partiellement différents) qui en découlent. Le plus classique des exemples est celui du dwo "universel", figure initiale de dwo qui est présente dans le culte sous la forme des masques de feuilles, et celui de ses nombreuses figures ultérieures (dwo de Patama, de Lebere, de Kwele...) ayant autels et masques personnels (de fibres).

A côté de ces "figures ultérieures" existent aussi des entités de même catégorie dont le culte a été acquis auprès de ceux qui en détiennent les droits. Ainsi, chaque communauté villageoise réserve un culte majeur à la figure primordiale de la brousse (sous le nom de kimi tâña kwè soxo : soxo commun du village), mais des cultes secondaires (et souvent privés) sont voués aussi à des soxo d'autres villages qui ont été adoptés (à Kurumani : soxo de Lèkoro ou Zyaǩo).

b) De simples autels, ou lieux, ou encore objets sacrés qui, bien qu'étant liés directement au culte d'une entité individualisée de

constitue un événement dont rend compte le mythe cosmogonique bobo, récit ordonné des manifestations premières successives des huit entités primordiales ci-dessus énumérées. Voir également à ce sujet GLM $1980: 120$ et 279 .

8. funanyone, à 1 a différence des autres termes énumérés, est un nom collectif. Il regoupe un grand nombre d'entités personnalisées mais qui sont de même nature spirituelle. 
TABLEAU I

\begin{tabular}{|c|c|c|c|c|}
\hline Catêgories & Noms & Type & $N^{\circ}$ & $\begin{array}{l}\text { Rapports } \\
\text { éventuels }\end{array}$ \\
\hline wuro & $\begin{array}{l}\text { wuro } \\
\text { wuro no tènè dỉbi }\end{array}$ & $\begin{array}{l}\mathrm{a} \\
\mathrm{a}\end{array}$ & $\begin{array}{l}1 \\
2\end{array}$ & \\
\hline $\begin{array}{l}\text { kwere } \\
\text { entité de } \\
\text { la forge }\end{array}$ & $\begin{array}{l}\text { kwere } \\
\text { tokwiyanô }\end{array}$ & a & - & $\begin{array}{l}\text { wuro-dwo } \\
\text { wuro- dwo }\end{array}$ \\
\hline 20 & $\begin{array}{l}\text { zo do } \\
\text { foxoba (zo) } \\
\text { katamâga (zo) } \\
\text { zo kền do }\end{array}$ & $\begin{array}{l}\mathrm{a} \\
\mathrm{a} \\
\mathrm{a} \\
\mathrm{a}\end{array}$ & $\begin{array}{l}4 \\
5 \\
6 \\
7\end{array}$ & \}wro \\
\hline $\begin{array}{l}\text { soxo } \\
\text { entités } \\
\text { tribales }\end{array}$ & $\begin{array}{l}\text { kixi tâna kwè soxo } \\
\text { soxo ha } \\
\text { lyako soxo } \\
\text { finiwala soxo } \\
\text { taane soxo } \\
\text { pô } \\
\text { syêsaprè } \\
\text { polo }\end{array}$ & $\begin{array}{l}\mathrm{a} \\
\mathrm{a} \\
\mathrm{a} \\
\mathrm{a} \\
\mathrm{a} \\
\end{array}$ & $\begin{array}{r}8 \\
9 \\
10 \\
11 \\
12\end{array}$ & 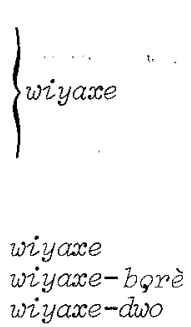 \\
\hline dwo & $\begin{array}{l}\text { Kweze dwo } \\
\text { dwosa } \\
\text { sibe dwo } \\
\text { patama dwo (foroba) } \\
\text { patama dwo (zakane) } \\
\text { kafuna zi } \\
\text { ko } \\
\text { flûtes ot tambours } \\
\text { sibe sine pati } \\
\text { forkôma pati } \\
\text { sibe dalza pati } \\
\text { tambours (griots) } \\
\text { (dwo-soxo): } \\
\text { dwo-soxo de dwosa } \\
\text { dwo-soxo de pat. dwo } \\
\text { aute1s du devin }\end{array}$ & $\begin{array}{l}\mathrm{a} \\
\mathrm{a} \\
\mathrm{a} \\
\mathrm{a} \\
\mathrm{a} \\
\mathrm{b} \\
\mathrm{b} \\
\mathrm{b} \\
\mathrm{b} \\
\mathrm{b} \\
\mathrm{b} \\
\mathrm{b} \\
\mathrm{c} \\
\mathrm{c} \\
\mathrm{c}\end{array}$ & $\begin{array}{l}16 \\
17 \\
18 \\
19 \\
20 \\
21 \\
22 \\
23 \\
24 \\
25 \\
26 \\
27\end{array}$ & 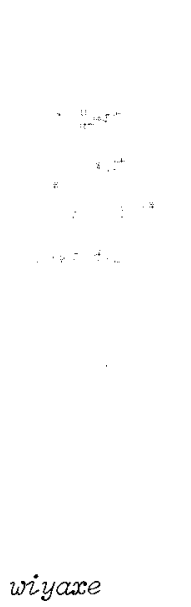 \\
\hline
\end{tabular}




\begin{tabular}{|c|c|c|c|c|c|c|}
\hline $\begin{array}{l}\text { funanyone } \\
\qquad . \\
\qquad\end{array}$ & $\begin{array}{l}\text { osöbeozô } \\
\text { môkasa } \\
\text { bèra } \\
\text { kumorasiri } \\
\text { sindo } \\
\text { sâtogo } \\
\text { zazawozo } \\
\text { (wiyase-funanyone): } \\
\text { duba sît } \\
\text { duba ña } \\
\text { vyetogo } \\
\text { do (intérieur) } \\
\text { do (extérieur) }\end{array}$ & $\begin{array}{l}a \\
a \\
a \\
a \\
a \\
a \\
a \\
c \\
c \\
c \\
c \\
c \\
c\end{array}$ & $\begin{array}{l}31 \\
32 \\
33 \\
34 \\
35 \\
36 \\
37 \\
\\
38 \\
39 \\
40 \\
41 \\
42\end{array}$ & $\begin{array}{l}\text { dwosa } \\
\text { dwosa } \\
\text { dwo }\end{array}$ & 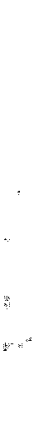 & $\therefore$ \\
\hline $\begin{array}{l}\text { wiyaxe } \\
\text { entités de } \\
\text { la chasse }\end{array}$ & $\begin{array}{l}\text { kiri tâna kwè w. } \\
\text { kunuma kwê } \\
\text { tokatsa } \\
\text { w. des Pyê ta koma } \\
\text { a/ foxoba tolo } \\
\text { b/ foxoba wiyaxa } \\
\text { wurosa } \\
\hat{n} a \hat{n} o \text { ma } \\
\text { nâko } \\
\text { dibi de nâko } \\
\text { bâkoro } \\
\text { dibi de bâkoro } \\
\text { dabu } \\
\text { wosini }\end{array}$ & $\begin{array}{l}\mathrm{a} \\
\mathrm{a} \\
\mathrm{a} \\
\mathrm{a} \\
\mathrm{a} \\
\mathrm{a} \\
\mathrm{a} \\
\mathrm{a}\end{array}$ & $\begin{array}{r}43 \\
44 \\
45 \\
46 \\
47 \\
- \\
48 \\
49\end{array}$ & $\begin{array}{l}\text { soxo } \\
\text { soxo } \\
\text { soxo } \\
\text { soxo } \\
\text { soxo } \\
\text { soxo, } \approx 0 \\
\text { soxo } \\
\text { soxo }\end{array}$ & : & 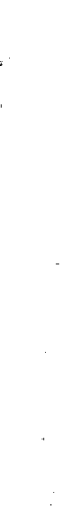 \\
\hline $\begin{array}{l}\text { entités } \\
\text { dérivées }\end{array}$ & $\begin{array}{l}\text { bqrè } \\
\text { saprè } \\
\text { saprazô } \\
\text { kiri } \\
\text { kiri kônaigö } \\
\text { küiye }\end{array}$ & $\begin{array}{l}\mathrm{a} \\
\mathrm{a} \\
\mathrm{b}\end{array}$ & $\begin{array}{l}56 \\
57 \\
58\end{array}$ & 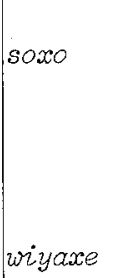 & & \\
\hline
\end{tabular}


type "a", sont l'objet également d'un culte personnel. C'est autant au nom de l'entité dont ils participent ( Wwo ou bgrè par ex.) qu'en leur nom propre que les tambours de dwo (23) ou que la terrasse du temple des ancêtres (sapro zô : 50) reçoivent des sacrifices et sont objets d'un culte.

c) Des entités qui procèdent à la fois de deux catégories : les wiyaxe-funanyone par ex. A ce cas on peut joindre des autels voués au culte conjugué de deux entités : dwo-soxo $(28,29)^{9}$

d) Des entités qui ont une individualité marquée et un culte personnel, mais dont on admet qu'elles dévivent originellement d'une autre entité, ce qui explique leur appartenance commune à une même catégorie. Les autels tribaux pô (13) syêsaprè (14) etc. dérivent de soxo et sont pour cette raison dans sa catégorie.

Dans le tableau 1 nous avons pris soin de faire référence au type, de "a" à "d", auquel se rattache chaque entité. Il faut cependant signaler qu'une entité, bien qu'appartenant d'une façon déclarée à une catégorie donnée, peut avoir au surplus certaines sortes de rapports avec une ou plusieurs autres catégories sans qu'il s'agisse véritablement d"une double appartenance comme dans le type "c". Ainsi, il existe un rapport conceptuel entre un petit esprit comme sindo et la grande divinité dwosa.; c'est pourquoi,à la différence des autres funanyone, sindo -- comme dwo -- refuse toute offrande, c'est aussi pourquoi il se voit sacrifier un animal hautement valorisé comme le bélier.

Les rapports éventuels d'une entité à une catégorie autre que la sienne ont été indiqués dans une colonne spéciale du tableau 1.

1.2. Le second de nos documents de base, en 1 'espèce 1 'organigramme des cultes, n'a subi qu'une seule modification notable. zo, hiérophanie aquatique, est apparu clairement en effet, au terme de nos enquêtes, comme ayant aussi une place de choix dans la liste des catégories spirituelles fondamentales ; jusqu'alors nous avions un peu mésestimé la place et 1 'importance des entités de la catégorie $z 0^{10}$, mais c'est

9. Les nombres placés entre parenthèses aussitôt après $1 \mathrm{e}$ nom d'une entité renvoient aux tableaux 1 et 3 .

10. GLM 1980 : 142 note 1 et 289 note 2 . Nous avions, en effet, fait 
de la nature même des sacrifices offerts qu' on est en droit de conclure, comme on le verra, à une position majeure de l'élément aquatique dans le concert universel.

\section{LA THEORIE}

Tandis que progressivement s'établissait la liste des entités et de leurs autels, nous procédions également à un examen approfondi des modalités de 1 'acte sacrificiel lui-même : nous appuyant sur 1 'abondante documentation en notre possession et sur de nouvelles observations, tentant aussi d'interroger le vocabulaire du sacrifice et de délimiter des champs sémantiques. Si l'on se reporte à l'article qui a rendu compte des résultats de ce travail ${ }^{11}$, on observe -- comme nous le rappelions ici même dès le début -- l'existence de constantes tant dans la nature des actes accomplis au cours du procès sacrificiel que dans le choix des matières végétales ou des espèces animales offertes, choix visiblement fait en fonction de leur nature ou de leurs caractères physiques.

Tout sacrifice comporte en effet, chez les Bobo, 1'usage bien précis de matières destinées aux offrandes et/ou de victimes animales destinées à être immolées.

Les matières obzatoires déposées sur les autels sont : 1a cendre, la bière de mil, l'hydromel, l'eau pure, l'eau mise en composition avec de la farine et donnant deux produits très différents : sêkèzè (pâte de petit mil, produit à consommer) ou fogo zo (farine de mil quelconque simplement délayée, prođuit non consommé).

De ces six matières ou produits, deux seulement ont une fonction pleinement identificatrice et possèdent donc un caractère de pertinence dans le cadre du code sacrificiel : sêkèzè et fogo zo ${ }^{12}$. Il est en effet

de zo un simple "esprit" (funanyonô). A ceci près que la place de zo doit être reconsidérée, tout ce que nous avons dit par ailleurs reste vrai, notamment la capacité de 1 'élément universel zo à s'individualiser et son rapport préférentiel avec la catégorie wiyaxe, génies possédant des droits sur le lit des cours d'eau qu'en des temps mythiques ils ont creusés.

11. GLM 1981.

12. Les symboles adoptés par nous pour ces deux produits sont les chiffres 1. (sêkèżè) et 2. (fogo zo). I1 convient de bien souligner que ces chiffres n'indiquent pas ici une hiérarchie de valeur (GLM 
admis que toutes les entités sans exception se reconnaissent au choix personnel qu'elles font dans les deux alternatives successives qui s'offrent à elles : accepter ou refuser le seul fait de recevoir une offrande et, dans le premier cas, prendre l'un des deux produits en question.

Les autres matières citées n'ont pas de place dans un code faute de posséder un pouvoir classificateur suffisant, les unes, comme la cendre, étant reçues indifféremment par toutes les entités, les autres n'étant acceptées ou refusées que par l'une d'entre elles seulement : ainsi pour l'eau pure réservée aux seuls ancêtres (b̧rê) ou pour la bière de mil ${ }^{13}$ acceptée par toutes à $1^{\prime}$ unique exception d'une figure de dwo (la triade des sibe - 16, 17, 18).

Les victimes animales sélectionnées pour leur grand degré de représentativité appartiennent aux espèces animales suivantes :

Poulets (P), Ovins (O), Caprins (C) .

D'autres victimes sont d'un emploi trop limité pour avoir été retenues : chat, chien, pintade, taureau...

Sont également pris en compte, outre 1'espèce, deux caractères physiques bien utiles pour provoquer des discriminations :

-- le sexe : mâle et femelle ( $\mathbf{\Lambda}$ et $\bullet$ ) ;

-- les couleurs du pelage ou du plumage : blanc (b), rouge ( $r$ ), noir (n), auxquelles s'ajoute la couleur dite indifférente (i) qui recouvre aussi les cas de couleurs à tons multiples ou mêlés ${ }^{14}$.

Ne prenant finalement en considération que les seuls éléments signifiants entrant dans le code sacrificiel bobo -- à savoir donc espèce, sexe, couleur de la victime et nature de l'offrande -- nous avons pu en venir à établir ce qui devait constituer la source principale de notre réflexion sur le sacrifice : le relevé complet des

1981 : 110). Le cas du refus d'offrande est figuré par le chiffre $\emptyset$. (le trait barrant le chiffre 0 ayant pour seul but d'éviter une confusion avec la lettre o majuscule signifiant "ovins").

13. L'hydrome1, quant à lui, ne sera pas retenu ici en raison de $1 \mathrm{a}$ grande variabilité de son usage : exclusif pour dibi de nâako (51); seulement possible et nullement nécessaire pour nôato (50) ; obligatoire (mais accompagné de bière de mil) pour les wiyaxe funanyone ; rigoureusement prohíbé pour dwo...

14. GLM 1981 : 113. 
TABLEAU 2

symboles et hiérarchies

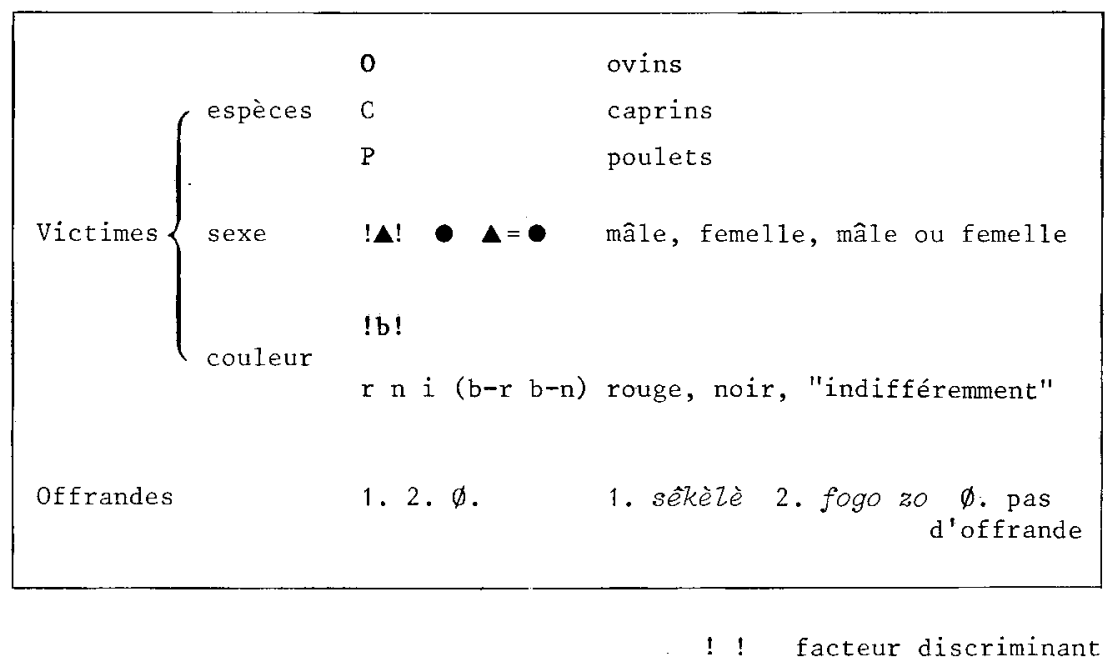

options faites individuellement par chacune des 61 entités spirituelles présentes dans notre 1 iste. Ce relevé, auquel nous nous référerons désormais, est présenté ici dans le tableau 3.

Avant même d'étudier dans le détail les options de chaque entité et de voir comment elles s'interprètent, il nous paraît nécessaire, pour la clarté de notre propos, de faire une première analyse des résultats du dépouillement du relevé et d'exposer les grands traits de la théorie que nous proposons et dont nous n'avons laissé jusqu'ici qu'entrevoir 1'existence.

Au seul examen superficiel du tableau 3 nous sont très vite apparus des ensembles d'options cohérents qui coincidaient, semble-til, avec les grandes catégories. Pour mettre en évidence de tels ensembles et pour concrétiser l'existence du code que I'on soupçonnait, nous avons utilisé les symboles dont ont été pourvues chacune des victimes ou matières sacrificielles sélectionnées pour leur caractère de pertinence (cf. tableau 2). Derrière le nom de chacune des entités nous avons donc placé une suite de symboles qui se présentent comme 
les "facteurs" d'une véritable formule, une "formule sacrificielle" résumant les exigences personnelles de l'entité en matière d'oblations et de victimes et exprimant, de façon figurative, toute sa singularité. Dans l'ordre, on a énoncé successivement : l'espèce de la victime, son sexe, sa couleur, la nature de l'offrande.

zo, par exemple, entité éponyme de la catégorie "Eau", demande le sacrifice des poulets $(P)$ de sexe quelconque $(\boldsymbol{\Delta}=\mathbf{0})$, de béliers ou de brebis ( 0 ), mais obligatoirement les uns comme les autres de couleur blanche (b) ; zo exige également une offrande de sêkèzè (1.). La formule sacrificielle de zo s'écrit :

\section{PO $\Delta=0$ b 1 .}

Cette formule diffère notablement de celle de soxo, l'entité éponyme de la catégorie "Brousse" qui veut des poulets et des caprins de sexe bien défini (mâle : $\boldsymbol{\Delta}$ ) et de couleur soit indifférente (i), soit rouge $(r)^{15}$ accompagnée d'une oblation de fogo zo:

$\mathrm{PC} \boldsymbol{\Delta} \mathrm{i}-\mathrm{r} 2$.

Si, comme nous l'avons fait, on établit en suivant cette méthode la formule sacrificielle de toutes les entités appartenant aux huit grandes catégories spirituelles, et si l'on se livre à l'exercice des comparaisons, on voit clairement s'affirmer les ensembles cohérents entrevus à la première lecture du tableau 3. Plus encore, c'est tout un jeu de rapports d'analogie ou d'opposition fonctionnels et donc pertinents qui se révèle.

Les modalités de ce jeu de rapports entre les formules ou entre les facteurs qui composent ces dernières s'organisent en une véritable combinatoire régie par un certain nombre de principes théoriques qu'il nous faut énoncer dès maintenant.

\subsection{Rapports pertinents entre facteurs}

Entre différents facteurs peuvent exister des rapports tels que si l'un des facteurs est retenu la présence de l'autre est entrainée nécessairement. Des couples se constituent ainsi qu'on retrouve dans

15. Le tiret placé entre les symboles de facteurs signifie "l'un ou 1 'autre selon $l^{\prime}$ espèce animale". Ici $i-r$ se réfère donc aux espèces dans 1 'ordre indiqué PC : victime de couleur indifférente pour 1 'espèce poulets, victime de couleur rouge pour l'espèce caprins. 
toutes les formules sacrificielles. Ces couples sont généralement placés en situation d'opposition pertinente.

a) Rapports entre espèce de la victime et nature de l'offrande.

Lorsque les ovins sont sacrifiés, l'offrande est obligatoirement 1. $\left(\right.$ sêkètè) ${ }^{16}$ :

01.

A l'inverse, lorsque des caprins sont sacrifiés, l'offrande est obligatoirement 2. $(\text { fogo zo })^{16}$ :

C 2 .

On a donc :

$$
01 . / C 2
$$

b) Rapports entre l'espèce et sa couleur.

Les ovins sacrifiés sont tous et toujours de couleur blanche ${ }^{17}$ :

$$
0 \mathrm{~b}
$$

Les caprins sont en bonne règle rouges; toutefois la couleur rouge est, comme on le verra (cf. 2.4.), en affinité avec le noir et même avec les couleurs dites "indifférentes", c'est à dire à tons multiples où le rouge est d'ailleurs souvent présent. La relation des caprins à la couleur est finalement :

$$
\text { C } \operatorname{rni}
$$

On a donc en opposition :

$$
\text { o b / c rni. }
$$

16. Sauf naturellement cas où l'entité refuse, à titre personne1, toute offrande.

17. Est propre au sacrifice tout mouton ( $g w a$ ) dont la toison est entièrement blanche, cas fréquent. Quelques taches discrètes peuvent être tolérées, mais si l'extrémité des quatre pattes est rouge et si 1 'animal "mange dans le rouge", i.e. si sa bouche est rouge, il est impropre au sacrifice. Si le mouton "regarde dans le rouge" (a l'entour des yeux rouge) et "marche dans le rouge" (pieds rouges), il est aventureux de le tuer et seul peut le faire un prêtre très expérimenté. Les moutons à cou rouge (muga ma pene) sont acceptés parfois (par b̧rè en particulier). Les moutons tout rouges (gwa pene) ou tout noirs ( $g w a g \hat{u}$ ) existent, mais sont évidemment totalement exclus des sacrifices puisque contraires au code.

Il faut enfin citer le cas exceptionnel du bélier para di gî ("joues noires") qui "mange, respire et regarde dans le noir" et dont 1'imnolation peut épargner une vie humaine si elle est menacée par suite d'une très grave transgression commise. 
c) Rapports entre sexe et couleur de la victime.

Signalons d'abord qu'est reconnue par les Bobo -- comme c'est fréquemment le cas en Afrique -- une correspondance entre le sexe de 1 'entité et celui de la victime qui lui est offerte. Nous verrons que 1 'appartenance sexuelle de certaines entités n'est pas toujours clairement établie ou que leur sexe peut être considéré comme un facteur de moindre importance (cf. infra 2.3.b), mais lorsque le sexe de l'entité est bien affirmé, celui de la victime doit lui correspondre impérativement.

Au plan des rapports entre les facteurs sexe et couleur dans la formule sacrificielle, le code prend en compte une symbolique des couleurs qui attribue des connotations opposées rouge pour un individu mâle et blanche pour une femelle. Le noir, bien qu'il ait, rappelonsle, un rapport au rouge, semble être plutôt marqué par le signe femelle. Quant à la couleur "indifférente", elle n'a pas de lien conceptuel avec un sexe quelconque.

on peut donc écrire :
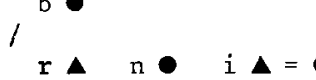

A) Rapports entre la couleur de la victime (ou son sexe) et la nature de l'offrande.

L'offrande 1., sêkèzè, est obtenue à partir d'un petit mil qui a été très soigneusement vanné, puis lavé et enfin finement pizé. La farine qui en résulte, délivre -- mêlée à de l'eau -- une pâte d'apparance très blanche. sêkèzè est donc associé dans $1^{\prime}$ esprit à la couleur blanche et à tout ce qui entre dans son champ symbolique, notamment les ovins et l'appartenance sexuelle femelle.

A l'opposé, l'offrande fogo zo relève de la couleur rouge et, partant, aussi de la couleur noire. Sa matière, petit mil ou souvent sorgho, n'est ni vannée ni lavée et seulement écrasée à la meule, ce qui lui donne effectivement une couleur brun-rouge. fogo zo rejoint ainsi dans la catégorie du rouge les caprins et l'appartenance sexuelle mâle.

A nouveau, on se trouve devant deux couples de facteur opposés : b $1 . / \mathrm{rn} 2$.

e) Notons pour terminer que les rapports espèce-sexe et sexeoffrande sont jugés non pertinents. 


\subsection{Enchainement naturel des rapports : formules types}

Si, tenant compte des rapports pertinents ainsi codifiés, on veut les enchaîner dans 1'ordre retent pour leur énoncé, deux formules bien distinctes se construisent. D'une part, on aura des sacrifices avec des ovins qui supposent couleur blanche de la victime et offrande de sêkèlè: o b 1., d'autre part, des sacrifices avec caprins qui supposent couleur rouge (ou noire ou indifférente) et offrande de fogo zo : C rni 2. En ajoutant lès rapports du sexe à la couleur, on obtient en définitive deux formules $\mathrm{F} 1$ et $\mathrm{F} 2$ dans lesquelles tous les facteurs sont opposés un à un :

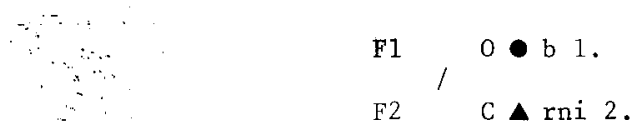

\subsection{Facteurs "discriminants"}

Théoriquement, toutes les formules sacrificielles des entités spirituelles bobo devraient renvoyer à l'une ou à l'autre de ces, deux formules types. Dans la pratique, cela n'est vrai qu'à la condition de tenir compte, par hypothèse, de certains aménagements introđuits dans le système très rigide d'opposition des facteurs. Ces aménagements permettraient une plus grande souplesse d'application et ouvriraient I'éventail du choix des formules tout en sauvegardant le principe affiché d'une bipolarité bien tranchée.

Des trois éléments signifiants retenus par le code sacrificiel -espèce, couleur, sexe -- seuls les deux derniers peuvent se voir attribuer une sorte de primauté. Le facteur de la formule sacrificielle sur lequel est ainsi porté 1 'accent se trouve alors doté d'une aptitude accrue à générer des distinctions ; nous dirons de ce facteur qu'il est "discriminant". Le poids du facteur "discriminant" est tel qu'en sa présence la pertinence de certains rapports peut être remise en cause.

a) Sur le plan de l'espèce

Remarquons que les poulets n'ont pas été jusqu'ici inclus dans nos formules sacrificielles. C'est qu'en effet 1'usage de poulets dans le sacrifice n'est guère pertinent en lui-même puisque tout sacrifice comporte obligatoirement la mise à mort d'un poulet au minimum. En fait, un seul des divers poulets qui peuvent être sacrifiés dans le cours du 
procès sacrificiel compte vraiment : celui qui est immolé au même moment que les ovins ou les caprins ${ }^{18}$. Or ce volatile doit être normalement en conformité de sexe et de couleur avec le quadrupède qu'il accompagne dans la mort. Si, par exemple, un poulet est tué avec une brebis, il devra, comme cette dernière, être du sexe femelle et de couleur blanche (cf. tableau $3: 17$ ) ; s'il accompagne un bouc, il sera mâle et de plumage rouge (tab. $3: 43$ ). Il est vrai qu'existent des sacrifices où les seules victimes sont des poulets. Ce cas recouvre des situations assez diverses et difficilement réductibles à une explication unique -nous y revienđrons en troisième partie. En règle générale donc, puisque les poulets se conforment le plus souvent aux règles qui fixent les rapports de pertinence entre les autres facteurs, rien n'empêche d'adjoindre régulièrement le symbole des poulets (P) à ceux des ovins ou caprins qu'ils accompagnent. Les deux formules sacrificielles types deviendront alors pour être complètes :

$$
\begin{array}{ccc}
\mathrm{F} 1 & \mathrm{P} 0 \bullet \mathrm{b} 1 . \\
\ldots \quad \ldots & \mathrm{F} 2, \quad \mathrm{PC} \operatorname{rni} 2 .
\end{array}
$$

Il reste que, en matière d'espèces, seuls les ovicaprins ont une fonction vraiment classificatoire. Pour autant on ne peut dire que des deux espèces 1 'une ait été dotée d'un caractère discriminant, dans le sens où nous l'entendons. Le choix d'ovins, ou au contraire de caprins, n'engendre rien d'autre que les simples rapports de pertinence édictés par le code avec les facteurs correspondants de couleur et de nature d'offrande.

b) Sur le plan de la couleur

Des différentes couleurs sélectionnées par le code sacrificiel, c'est le blanc qui a été retenu comme facteur discriminant.

Lorsque ce facteur "coulèur blanche" (b) est présent dans une formule, ses rapports à l'espèce et à la nature de l'offrande subsistent, mais son rapport au sexe perd toute pertinence. Ainsi, la

18. GLM 1981 : 113. Les autres poulets sacrifiés ne sont pas directement en rapport avec 1 'objet principal du sacrifice (ils sont simplement immolés à l'intention des participants), on est donc moins exigeant sur leurs caractéristiques. Ces poulets ne sont retenus pas nous dans l'énoncé de la formule sacrificielle. 
couleur blanche entraîne toujours le choix d'un ovin (cf. 2.1.b) et l'ovin, tout autant que la couleur blanche, mène au choix de l'offrande sêkèzè (2.1. a) ; on retrouve bien, conformément au code :

$\mathrm{PO}-\mathrm{b} 1$.

En revanche, le rapport sexe-couleur, tel qu'il est fixé par le code, n'est plus appliqué, le blanc ne s'associe plus nécessairement à une victime de sexe femelle et peut désormais devenir la couleur d'un màle (bélier en 1 'occurence).

Quand le blanc est discriminant, on peut donc aussi bien trouver :

$$
\text { PO b } 1 \text {. }
$$

que :

$$
\text { PO } \Delta \text { b } 1 \text {. }
$$

Ou bien l'entité est vraiment indifférente au sexe de la victime qu'on lui offre -- on prend celle dont on dispose -- ou bien on tient compte du sexe de l'entité et on lui destine une victime de sexe correspondant. Si nous utilisons le symbole $\Delta=0$ pour signifier "mâle ou femelle indifféremment ou suivant le sexe de l'entité", la formule type 1, rectifiée, se présente définitivement ainsi :

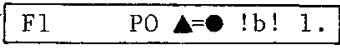

Pour marquer son caractère discriminant, le symbole du facteur sera encadré de deux points d'exclamation.

c) Sur le plan du sexe

A la formule 1 , où le facteur sexuel est tenu en moindre compte, répond une formule dans laquelle c'est, à l'inverse, sur le sexe -- et préférentiellement mâle -- qu'est mis l'accent. Comme il est naturel (cf. 2.1.c), le facteur mâle n'est ainsi affecté d'un caractère discriminant que dans les formules sacrificielles attribuées à des entités dont l'appartenance sexuelle mâle est elle-même considérée comme étant un trait dominant de leur personne.

Le sexe mâle entraîne par le jeu des rapports pertinents théoriques, le choix d'une victime rouge -- en 1 'occurence donc un bouc -et ce caprin, tout autant que la couleur rouge, conduit, de son côté, à faire 1'option de fogo zo en matière d'offrande. On retrouve de cette manière la formule $\mathrm{F} 2$ avec pour seul ajout le signe indiquant 
le facteur discriminant :

$$
\text { F2 PC !A! rni } 2 \text {. }
$$

Cette formule convient, avons-nous dit, à toute une série d'entités qui ont en commun d'être masculines, mais que se passe-t-il lorsqu'une entité femelle est classée dans 1'une de ces catégories à dominante mâle et dont les victimes sacrificielles désignées sont des boucs rouges?

Dans cette éventualité, il semble que les Bobo aient élaboré trois cas de figure.

- Premier cas : immolation d'une chèvre rouge (cf. tableau 3 : $18,26)$.

Le caractère mâle étant discriminant, il tendrait à figer les divers rapports pertinents qui restent dès lors en l'état. Le sexe femelle de la victime s'inscrit dûment dans la formule, mais il n'est plus en relation normale de pertinence avec la couleur. On se trouve devant la formule suivante qui reproduit simplement la formule F2 en inversant seulement le facteur sexe :

$$
\mathrm{PC} \odot x .^{19}
$$

Cette formule est logique -- au-delà des apparences -- si 1 'on considère le caractère mâle comme immanent et restant donc inscrit comme en filigrane. Il faudrait en conséquence écrire :

$$
\mathrm{PC}(! \Delta !) \odot \mathrm{r} \emptyset \text {. }
$$

- Deuxième cas : immolation d'une chèvre noire (cf. tableau 3 : $37,39$ et surtout 51,52$)$.

En se fondant sur le fait que, d'une part, le noir a un rapport de substitution et donc d'équivalence avec le rouge (faute de noir, on peut prendre du rouge : $n=r$ ) et, d'autre part, qu'il y a dans la symbolique du noir une constante féminine $(\mathrm{n}=)^{20}$, on peut, quand l'entité

19. Pour de toutes autres raisons -- elles sont de la catégorie dwo -il se trouve que les entités qui optent pour cette formule refusent 1 'offrande quí -- théoriquement -- devrait être ici 2.

20. Notons que les victimes sacrificielles noires sont toujours des femelles (sauf dans le cas très particulier des chats). Jamais on ne sacrifie des boucs noirs ou des moutons noirs. En matière de poulets l'usage de coqs noirs nous est signalé mais comme ingrédient de remèdes. 
est femelle, substituer au facteur mâle de la formule F2 le facteur femelle aussi bien que le facteur rouge au facteur noir et cela sans que la présence des autres facteurs soit mise en cause ( $P, C$ et 2.).

A nouveau on retrouve $F 2$ avec simple substitution de 0 à $A$ et de n à r (même remarque que dans la note 19 à propose de l'absence éventuelle d'offrande, la raison en pouvant être différente).

On a donc :

$\mathrm{PC} \bullet \mathrm{n} 0.2$.

Ou plus exactement, comme dans le premier cas :

PC (!)!) $\mathrm{n} \emptyset .2$.

On peut en conclusion considérer qu'en cas de victimes femelles lá formule F2 accepte l'existence d'une variante qui peut s'écrire, en résumant les deux cas décrits ci-dessus, de la façon suivante:

$$
\text { F2 bis } \quad P C \quad(! \Delta !) \quad r, n \quad \emptyset: 2^{21}
$$

- Troisième cas : immolation d'une brebis blanche.

Un raisonnement tout opposé aux précédents peut aussi être tenu, quoiqu'il semble parfaitement paradoxal puisqu'il mène à une inversion de tous les facteurs et à l'immolation d'une brebis blanche.

Si I'on considère en effet que I'accent est porté, dans les formules du modèle F2, sur le sexe de la victime, on peut juger que le rapport au sexe de l'entité elle-même devient de ce fait plus impératif que jamais. Poussant dès lors la logique du code jusqu'à son terme, on peut, ayant donné à 1 'entité femelle une victime femelle, faire jouer le rappprt normal sexe-couleur en immolant une victime blanche, c'est à dire un ovin, ce qui entraîne le choix d'une offrande de sêkè̉zè. C'est ainsi qu'on peut retrouver, dans une catégorie où les entités sont en majorité mâles et destinataires de formules sacrificielles du modèle F2 (boucs rouges), des entités femelles auxquelles s'adressent des sacrifices du modèle Fl (brebis blanche) :

$0 \circ 1$.

Dans ce cas précis - réduit il est vrai à trois entités de la catégorie wiyaxe (tab. $3: 44,45,47 \mathrm{~b}$ ) -- 1e système établi semble

21. Le symbole "n", précédé d'une virgule, évoquera, pour les entités se rattachant à la formule F2, l'éventualité d'une victime de couleur noire si le sexe de l'entité est femelle. 
être mis en défaut. A cela les Bobo ne nous ont fourni aucune justification acceptable, mais peut-être pouvons-nous émettre néanmoins une hypothèse.

La faille du raisonnement se produit au moment où, ayant une victime femelle, on choisit la couleur blanche alors qu'on pourrait, dans le cadre de la formule 2, prendre le rouge en dépit du code -cas 1 -- ou, mieux encore, le noir, ce qui est conforme au code -- cas 2 .

Le facteur "blanc" est, on le sait, déjà surévalué puisque discriminant, mais on peut se demander s'il ne jouit pas au surplus d'une prééminence sur tous les autres facteurs, y compris le facteur mâle quand il est lui-même discriminant. En présence d'une victime femelle, on aurait ainsi la tentation de prendre le correspondant couleur du plus haut niveau -- le blanc plutôt que le rouge ou le noir ; d'autant plus que cela annulerait du même coup l'effet mâle discriminant. Cela pose tout le problème de la hiérarchie éventuelle des facteurs.

\subsection{Hiérarchies}

Les divers facteurs entrant dans la composition des formules sacrificielles ne se présentent nullement comme un ensemble de termes égaux. En effet, un certain nombre de séries ordonnées sont soit clairement désignées par les instances religieuses, soit facilement déduites des faits. Si l'on semble donc pouvoir parler ici de hiérarchies, on ne s'autorisera cependant à le faire qu'avec prudence et sans préjuger de la nature vraie du rapport de subordination existant entre les facteurs énumérés. Ce n'est pas en termes de supériorité pure et simple que s'expriment les Bobo lorsqu'ils situent êtres et choses les uns par rapport aux autres. Bien souvent, plutôt que de rangs, il s'agit de positions relatives, de situations en des "lielix" -- au sens topologique du mot -- différents, mais non pas nécessairement de qualité "supérieure" ou "inférieure".

Dans le domaine des espèces vouées aux sacrifices, les ovins occupent sans conteste une place privilégiée, nous l'avons largement démontré ${ }^{22}$, tout en soulignant qu'en comparaison les caprins jouissaient

22. GLM $1981: 115$. 
d'une moindre estime. Par rapport à ces quadrupèdes, les poulets sont en position subalterne, mais cela semble être dụ̂ plus à la banalité de leur emploi qu'à une réelle infériorité de nature. On peut cependant écrire dans 1 tordre :

$$
\begin{array}{ll}
1 & 0 \\
2 & 0 \\
3 & P
\end{array}
$$

ou encore :

En matière de couleurs, il y a, nous $1^{\prime}$ avons constaté ici même, une très forte valorisation du blanc et cela en raison notamment des symboles qu'il véhicule (lien au Dieu suprême, clarté, vertu, douceur, féminité, etc.). La couleur rouge se classe à 1 'opposé et dans un monde de symboles dotés de mérites moindres (violence, sexualité, dureté, sang...).

Le noir, quant à lui, est "ailleurs". Sa relation au rouge n'est pas de subordination, il y a même sur le plan hiérarchique-- nous l'avons vu -- une sorte d'équivalence, mais le vrai domaine du noir est très spécifique : c'est celui de $1 \mathrm{a}$ maladie, du mal sous toutes ses formes, c'est celui de tout ce qui échappe à la connaissance ; secret donc, secret de 1'initiation, secret des fautes carhées. Le noir est aussi le domaine de la brousse secrète, sauvage et dangereuse et, par voie de conséquence, c'est le monde de la chasse et des chasseurs. Curieusement, le noir, par le biais du ciel orageux, évoque aussi la pluie et, par des détours de pensée encore plus subtils où se retrouvent associées les idées de fécondité (eau, pluie), de maladie (règles) et surtout de secret (mystères de la génération), le noir connote enfin une certaine image de la féminité, très révélatrice de la psychologie bobo.

Les couleurs mêlées relevant de 1'option dite "couleur indifférente" (plumage du poulet) ont un contexte assez flou, elles présentent en fait, le plus souvent, un assortiment de blanc avec du rouge ou du noir (poulets gwenderi et kaba ma pene), mais ce sont plutôt ces deux dernières nuances qui semblent être retenues au plan de 1a signification.

Au total, s'il est vrai qu'une véritable prééminence est accordée au blanc, toutes les autres couleurs doivent être considérées comme 
étant de même rang :

$$
\begin{array}{lll}
1 & \mathrm{~b} \\
2 & \mathrm{rni} & \\
\text { (br bn) }
\end{array}
$$

Pour ce qui concerne le sexe, nul doute que le pouvoir soit acquis aux mâles dans 1 a société bobo, et cela aussi bien sur le plan religieux que social et économique. Il convient cependant d'être, pour le sujet qui nous préoccupe, très circonspect. Les vertus de la féminité sont si hautement valorisées qu'elles sont associées dans le code sacrificiel à la couleur blanche, elle-même dotée d'une réelle primauté puisqu'on lui accorde une fonction "discriminante". Du point de vue symbolique, mâles et femelles, s'ils restent profondément opposés de nature, ne peuvent donc être avec certitude placés à des niveaux hiérarchiques différents.

S'agissant enfin de la nature des offrandes, nous avons déjà souligné (cf. supra note 11) qu'aucune distinction hiérarchique n'était attribuée au fait d'offrir du sêkètè (arbitrairement coté par nous 1.), du fogo zo (2.) ou même de ne rien offrir du tout ( $\emptyset$ ). Tout en plaçant donc ces options sur le même rang, nous ne dissimulerons pas qu'existe -- sans qu'il soit clairement formulé -- le sentiment d'une certaine prééminence de l'offrande sêkèlè due au simple fait qu'elle s'associe aux deux seuls facteurs dotés d'une primauté reconnue : 1'espèce ovine et la couleur blanche.

Les dispositions hiérarchiques attachées aux divers facteurs affectent naturellement les formules que ces derniers composent. Si $l$ 'on rassemble selon leur ordre tous les facteurs, 0 et b viennent en position première, tandis qu'au second rang se placent $C$ et rni.

Dès lors, tenant compte des autres rapports de pertinence qui caractérisent chacun de ces facteurs ( 0 à $1 ., \mathrm{C}$ à $2 ., \mathrm{b}$ à $\bullet, r$ à $\mathbf{\Lambda}$ ), on retrouve les deux formules sacrificielles type dans un ordre qui, s'il reste conforme à celui retenu jusqu'ici, n'est plus seulement justifié par les besoins de l'énumération. L'ordre dans lequel se rangent les deux formules semble bien consacrer en effet l'idée qu'entre chacune des deux séries d'options sacrificielles existe ce qu'il faut bien appeler une hiérarchie, même si ce terme -- comme nous l'avons déjà souligné -- appelle quelques réserves. 
Au stade purement théorique où nous sommes, il semble difficile d'aller plus loin, ne serait-ce que par manque d'informations sur des points essentiels comme la nature des critères qui définissent les modalités de chacune des deux options et surtout l'identité précise et le caractère personnel des entités qui exercent des choix communs.

I1 convient donc de renouer sans plus tarder avec la pratique et de confronter notre théorie avec la réalité des cultes telle qu'elle transparaît dans le relevé du tableau 3. On le constatera, variantes, cas particuliers et exceptions ne manquent pas, mais les raisonnements tenus pour justifier ces diverses anomalies sont souvent plus riches d'enseignement que les termes abstraits de la théorie, toujours trop réducteurs.

\section{L'APPLICATION}

3.1. wuro, ta divinité suprême

\section{$P \wedge ! b ! 2$.}

Lès Bobo, à la différence de tant d'autres populations africaines, réservent un culte actif au Dieu suprême. L'autel de wroro, borne de banco cylindro-ogivale, ne reçoit des sacrifices sanglants qu'une fois l'an. C'est I'occasion de constater que wuro, divinité omnipotente, est à 1 a fois mesuré dans ses exigences et apparemment peu soucieux des normes sacrificielles qui s'appliquent plus strictement aux autres entités spirituelles.

C'est un simple coq que $1^{\prime}$ 'on offre à wuro, mais il est vrai qu'impérativement il doit être blane. La couleur blanche est 1'emblème de wuro et seront vouées au blanc toutes les entités participant plus ou moins directement de sa personne. On comprend dès lors les raisons de la primauté accordée par le code à la couleur blanche et du caractère "discriminant" qui lui est attribué.

Cela étant, force est de constater que, contrairement aux principes mêmes du code, les conséquences qu'entraîne le choix du blanc -à savoir un sacrifice d'ovin et une offrande de sêkèzè -- paraissent être négligées puisque c'est un unique poulet et une offrande de fogo zo que 1 'on réserve à wuro.

Pour justifier cet état de fait, les autorités religieuses 
$+\quad+1^{\prime}$ un et $l^{\prime}$ autre

$+(+)$ l'un ou $l^{\prime}$ autre - (+) par dêfaut ou en second

$+=+$ l'un ou 1 'autre, indifféremment ou suivant sexe de 1 'entité

- $\quad$ absence totale d'offrande

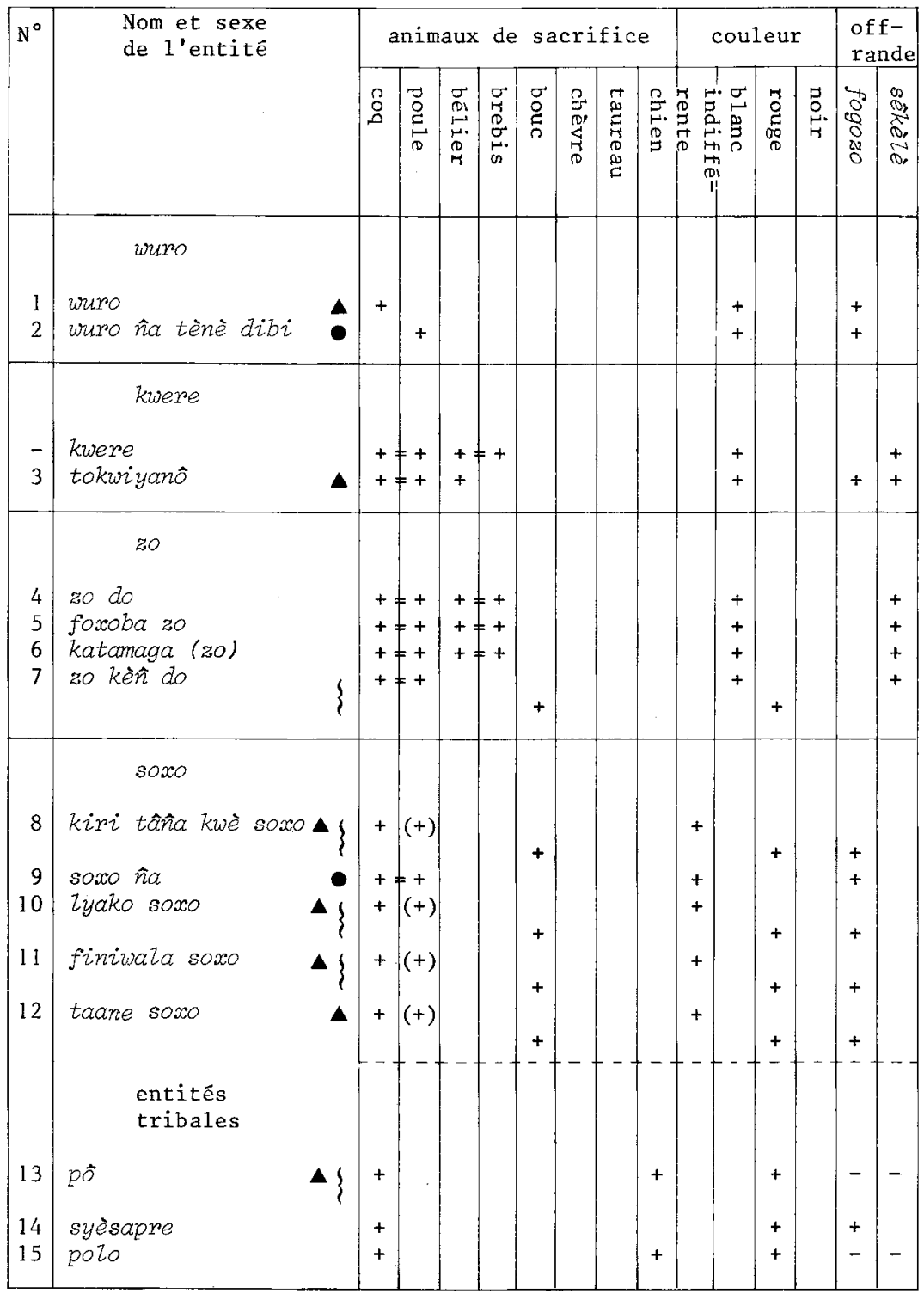




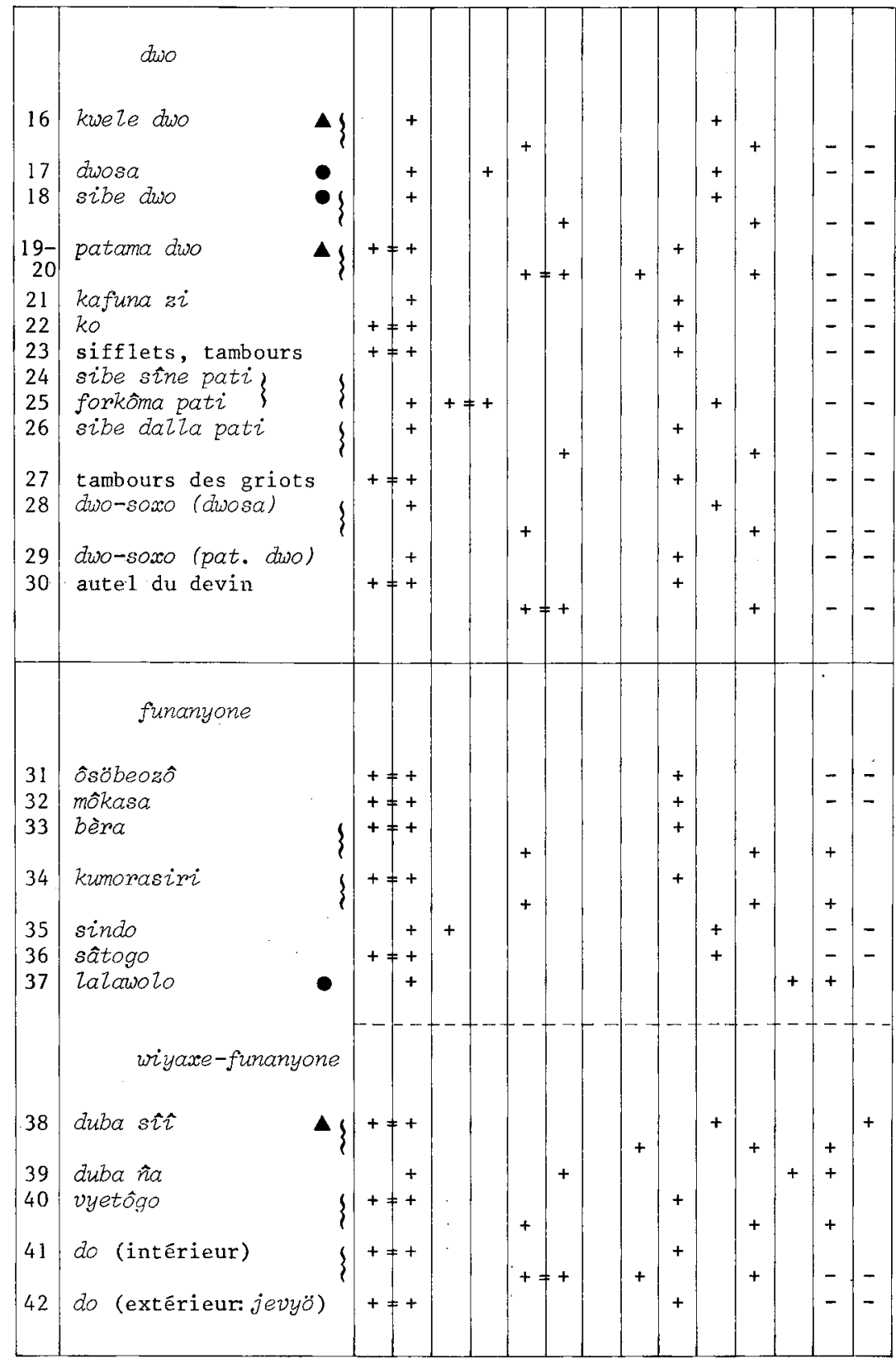




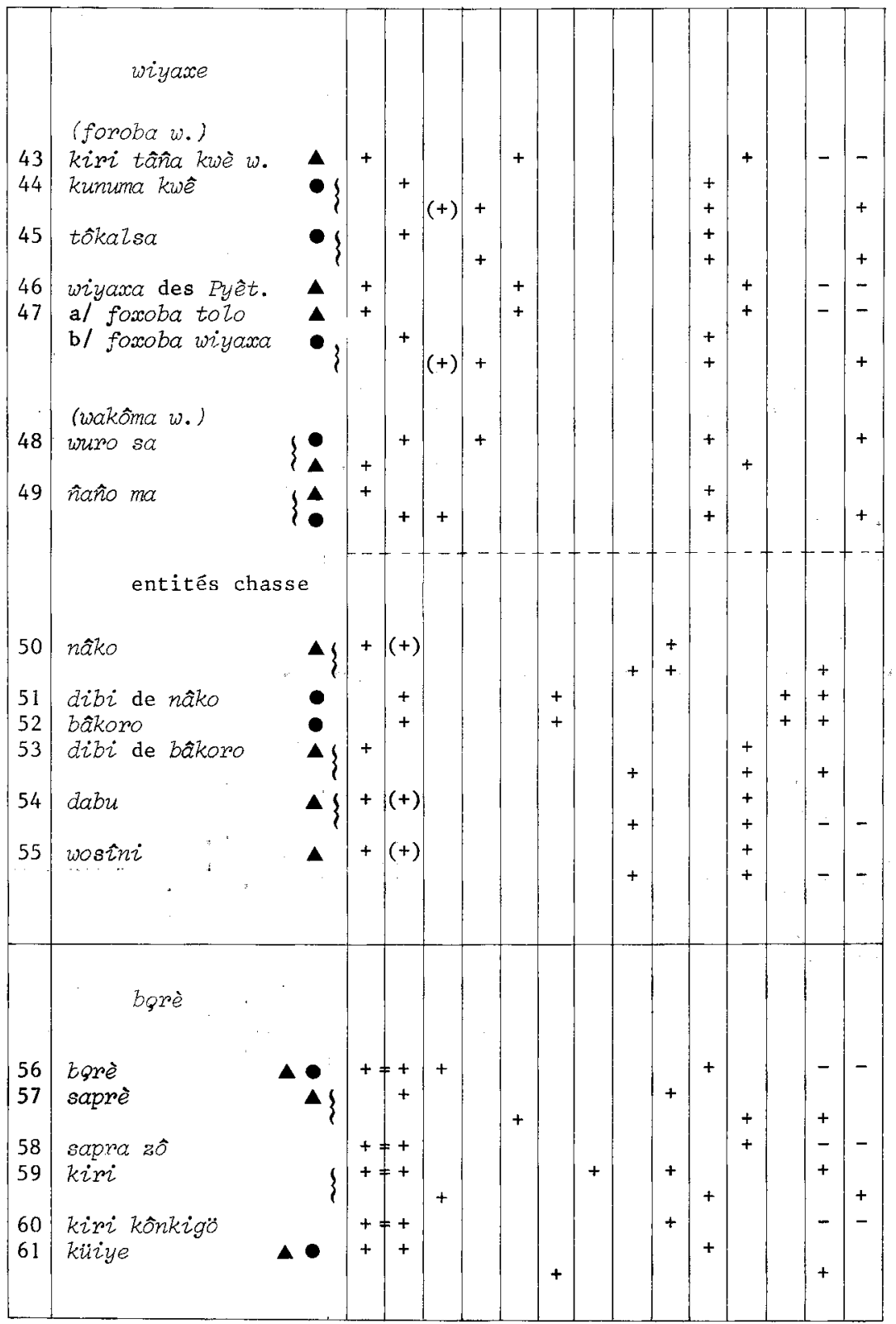


usent de trois arguments :

- Créateur de toutes choses, urro est indifférent aux biens terrestres dont il est de toutes façons le seul propriétaire. Point d'autre nécessité donc qu'un geste sacrificiel réduit à sa plus simple expression matérielle, poulet et petite offrande ou, autre exemple, quelques modestes galettes à l'occasion de ce rite également voué au Dieu suprême qu'est le salka wiyè dâga (GLM 1980: 113).

- Le choix d'un simple poulet en lieu et place d'un mouton se justifie par le principe en vertu duquel il est possible de substituer une chose de moindre valeur à ce qui est de haut prix lorsqu'au moins un caractère important leur est commun ( 1 a couleur blanche ici) ${ }^{23}$ : en somme, un poulet blanc = un mouton blanc, si le destinataire n'est pas trop exigeant...

- Quant à l'écueil que constitue 1 e don de fogo zo, tout à fait contraire aux conventions, il est assez habilement contourné par certains puristes qui choisissent du petit mil et le pilent légèrement au lieu de l'écraser afin de rapprocher, par cette technique ${ }^{24}$, fogo zo et sêkèlè et de le rendre compatible avec la couleur blanche.

Pour ce qui concerne le sexe mâle de la victime, nous savons qu'avec le discriminant "couleur blanche" la conformité avec le sexe de l'entité destinataire est respectée (2.3.b). Cela confirme le caractère mâle de wuro et justifie à l'inverse le poulet femelle -- blanc lui aussi -- destiné à wro ha tenè dibi (2), la "pierre (d'autel) de tenè, la femme de Dieu", une entité qui occupe d'ailleurs une place assez marginale dans les croyances bobo.

Au total, la formule sacrificielle de wrro n'est point si déviante qu'il apparaissait en première lecture.

3.2. kwere, $2 \alpha$ foudre

PO $\Delta=\bullet$ ! b! 1 .

kwere est une émanation directe de la puissance de wuro, son

23. Cf. in GLM 1981 : 114 , 1e cas du poulet degeseni que l'aspect de ses plumes reıd équivalent à un mouton.

24. Ce produit en quelque sorte intermédiaire est appelé tugo fogo zo. 
culte est "privé" (zakane) dans tout le nord du pays bobo parce qu'il est l'apanage du clan Gyoma (python) ${ }^{25}$, mais il est communautaire (foroba) dans le sud.

Participant de wroro, kwere exige le blanc et, par suite, ce sont des ovins et du sêkèlè qui lui sont dus. Les victimes peuvent être des femelles mais sont plus souvent mâles (béliers notamment).

tokwiyanô (3), 1'enclume (ou, par extension, la forge), entretient en tant qu'autel et objet majeur du culte pour les forgerons, un triple rapport avec kwere ${ }^{26}$, dwo ${ }^{27}$ et, par voie de conséquence, uriro. L'enclume, d'une part, est la pièce maîtresse de la forge -- lieu où opère le feu, élément né, selon les mythes, de kwere, la foudre. D'autre part, dwo a, comme uriro dont il procède, la foudre pour arme, mais il est aussi lié à l'enclume par le fait que s'y élaborent, sous l'effet du feu, le fer du rhombe et celui de la hache servant à tailler le masque -- rhombe et masque étant; rappelons-le, les formes sensibles sous lesquelles se manifeste dwo.

La formule sacrificielle de tokwiyanô (poulets blancs de sexe indifférent et ovins, mais mâles) dérive directement de celle de kwere avec néanmoins une anomalie unique en son genre dans le code bobo : les deux offrandes, sêkèzè (normalement entraînée par le facteur couleur blanche), mais aussi fogo zo, sont conjointement réclamées par tokwiyanô. Cette présence de fogo zo n'est pas toujours clairement explicitée, mais -- renforcée dans sa signification par le caractère mâle, assez accusé, de 1 'entité forge -- elle suggère que cette dernière ne relève pas seulement du domaine symbolique du blanc, mais aussi de celui -- tout opposé -- que circonscrit le rouge ; une couleur en rapport, on le sait, avec le sexe mâle et l'offrande fogo zo tout autant qu'avec les caprins (espèce dont des cas d'immolation sur l'enclume sont d'ailleurs quelquefois attestés).

L'ambivalence airisi démontrée de l'enclume sacrée explique peutêtre pourquoi les forgerons, maîtres du feu, peuvent évoluer impunément

25. A Kurumani, kwere ne possédant pas d'autel propre (celui-ci se trouve dans un village voisin, à Kyeba, chez les Dao Koroma Kôma qui 1'ont acquis des Gyoma) n'a pas été affecté d'un numéro d'ordre dans le tableau 3. 26. On peut invoquer kwere en frappant sur l'enclume.

27. Tout grand sacrifice sur 1'autel de dwo doit être précédé d'un sacrifice sur 1 'enclume. 
dans les sphères antagonistes du cosmos et agir tant en brousse qu'au village, tant en matière de nature qu'en matière de culture.

\section{3. zo, l'élément aquatique}

\section{PO $\boldsymbol{A}=\mathbf{O} ! \mathrm{b} ! \mathrm{l}$.}

30, entité d'ordre majeur, est aussi proche de wuro que kwere, elle est de plus le premier créé des éléments constitutifs de la terre.

Comme à kwere.sont donc offertes à zo des victimes de couleur blanche et d'espèce ovine accompagnées d'offrandes de sêkèzè, ce qui est parfaitement conforme aux termes de la formule 1. Cette formule laisse toute liberté pour le choix du sexe de la victime et on peut en effet voir des brebis ou des poules sacrifiées sur les autels de zo, mais une préférence assez nette est marquée à l'égard des ovins mâles. Certes, le sexe de zo, pas plus que celui de kwere, n'est jamais clairement précisé, mais une appartenance mâle est assez naturellement induite du rapport de ces deux entités à wrro.

Ce sont ainsi des béliers blanc qui sont immolés, non seulement à Kurumani sur trois des autels de 1 'entité zo (4, 5 et 6), mais aussi sur tous les grands autels du culte de l'eau qui rassemblent de nombreux fidèles à l'échelle ethnique (autel da fra près dè Bobo Dioulasso ou zoyoxo près de Bura) ou même interethnique (mare de kwa en pays bwa).

Seule fausse note dans cet accord quasi général, le cas très particulier de zo kèn do (7). Ill s'agit en l'espèce d'une transposition de 1 'autel de katamâga (6). En tant qu'aute1 de l'eau on lui destine un poulet blanc, mais en tant qu'autel situé dans le secteur "brousse" de 1 'espace (soxo) on lui offre, dans certains cas particuliers, des boucs rouges. La contradiction n'en est pas moins indiscutable.

\section{4. soxo, Za brousse}

$$
\text { PC } ! \mathbf{A} ! \text { i-r } 2 \text {. }
$$

Bien qu'étant compté au nombre des entités majeures et considéré, au même titre que kwere ou dwo, comme un fils de wuro, c'est à dire comme une part composante de sa personne même, soxo n'est pas placé sous le signe du blanc, la couleur de uuro. Tout au contraire, sowo, dont le caractère mâle est retenu comme facteur discriminant ${ }^{28}$ est assujetti aux 
termes de la deuxième formule type. La victime sacrificielle privilégiée de soxo est en effet le bouc rouge; doublement mâle, si l'on peut dire, à la fois par son sexe et par sa couleur, laquelle, on le sait, est en relation pertinente avec 1'appartenance mâle. Par voie de conséquence, s'impose 1 'offrande de fogo zo, matière "rouge", ellemême en rapport direct, selon le code, avec les caprins.

soxo demande également des coqs, néanmoins il peut accepter des poules par défaut ou dans le cas de sacrifices mineurs. La couleur des poulets est, ce qui peut étonner, jugée "indifférente". En réalité, ce sont les poulets à plumage unicolore qui sont contre-indiqués ; sont en revanche expressément réservés à soxo : le poulet bini nane aux couleurs tirant à la fois sur le rouge et le blanc; les poulets gwenderi, rayés rouge et blanc (ou noir) et surtout le poulet kaba ma pene, blanc taché de rouge à la naissance des ailes.

On observera que la couleur rouge est présente dans le plumage de tous ces poulets, ce qui confirme que, bien que déclarés de couleur "indifférente", les poulets de soxo sont néanmoins "du côté" du rouge. Dans le tableau des entités de la catégorie soxo, objets d'un culte à Kurumani, les formules sacrificielles sont pour la plupart conformes à la règle établie. Cela est vrai tant pour le soxo de. Kurumani même ( 8 : kimi tôna kuè soxo, "kimi autour duque1 on est assis", i.e. soxo commun du village) que pour les soxo originaires d'autres villages (Lyako ou Lèkoro, 10 ; Finiwala, village disparu, 11 ; taane soxo, soxo de Bura, 12) et objet pour ceux qui les ont amenés à Kurumani, d'un culte seulement privé.

Quelques commentaires s'imposent néanmoins à propos du soxo de sexe femelle (9) associé au soxo mâle de Kurumani (8). L'autel principal d'un soxo est toujours en brousse quoique non loin du village ; c'est là que l'entité est présente sous son aspect mâle prépondérant, mais soxo possède aussi une "épouse" ( $\hat{n} \alpha)$, d'importance mineure, dont 1 'autel est situé à l'intérieur du village, au fond du temple du 1ignage fondateur (sapra vyö). De petits sacrifices de poulets $y$ sont faits.

soxo ña étant une femelle peut recevoir, ce qui est normal, une

28. Noter que jamais une femme ne s'approche de 1 'autel de soxo, jamais une femme ne consomme la chair des sacrifices à lui destiné. 
poule, mais telle est forte la marque du mâle qui accompagne tout ce qui se rapporte à soxo-la-Brousse que soxo $\hat{n} \alpha$ peut aussi recevoir des coqs. Ainsi, alors que sur les autels du grand soxo mâle les poulets femelles ne sont offerts qu'à défaut de coqs -- $\mathbf{\Delta}(\mathbf{\bullet})$-- sur I'autel de la femelle coqs et poules sont sacrifiés indifféremment :

Autre différence, beaucoup plus marquante : pas de caprins immolés sur soxo $\hat{n} \alpha$. De ce fait n'est pas soulevé le problème évoqué ici en 2.3.c et relatif aux caractéristiques des quadrupèdes offerts aux entités femelles relevant de la formule de type 2. Néanmoins, sachant que les poulets de couleur "indifférente" sont, dans la catégorie soxo, jugés comme étant "du côté" du rouge, on peut dire que soxo ĥa se rattache au premier cas de figure : c'est une chèvre rouge qu'elle recevrait si elle avait droit aux caprins. Nous en avons la preuve, tout au moins pour ce qui est du choix du rouge, car il existe à Kurumani un second soxo du village, non répertorie ${ }^{29}$, dont 1 'autel femelle reçoit des poules sacrificielles rouges.

A propos des caprins, il convient enfin de noter que leur choix, comme victimes sacrificielles désignées pour les entités de la catégorie soxo, s'explique à coup sûr par le symbolisme qui s'attache à cette espèce (surtout lorsqu'elle est de surcroît rouge et mâle), mais également par la position subalterne qu'ils occupent dans la hiérarchie des espèces. Certes, soxo est une force redoutable qui plane sur un espace libre -- la brousse -- ouvert aux violences viriles de la vie sauvage, mais soxo, malgré cela et peut-être à cause de cela, est moins haut placé que lès entités qui relèvent, avec wuro, du blanc et des prestigieux ovins. C'est aussi pour cette raison qu'à soxo ne reviennent que des caprins.

L'opposition des formules sacrificielles de zo et de soxo connote une différence profonde de nature entre les deux entités et les deux éléments qu'elles représentent, mais cette opposition est lue aussi par les Bobo comme 1'illustration d'une chronologie et d'un ordre universel immanent : 1'eau précède et conditionne la vie végétale -- I'herbe dépend de la pluie ...

29. Pour satisfaire à 1 a demande de nos informateurs, ce soxo n'a pas été situé sur la carte et donc répertorié. c'est un lieu sacré très secret et très redouté qui marque le second des emplacements successifs anciens du village. 
Retenons à cette occasion que la présence de caprins dans une formule sacrificielle peut s'expliquer par la nature de l'entité destinataire, mais qu'elle est souvent aussi une façon de signifier son caractère un peu subalterne.

\subsubsection{Entités tribales $\quad$ P $! \mathbf{\Delta} ! \mathrm{r} \emptyset$.}

Les entités sur lesquelles se fonde le lien mystique qui définit ces groupements politico-religieux que nous appelons "tribus" (pô wrru taze) dérivent directement de la catégorie soxo. pô, l'entité éponyme de cette sous-catégorie (13), couvre en effet de son autorité de vastes fractions de 1 'espace brousse (domaine de soxo donc) où sont rassemblées les demeures de tous les hommes qui se reconnaissent solidaires dans le culte de I'une ou 1'autre de ses figures particulières ${ }^{30}$.

pô, c'est aussi l'arc, simple objet matériel servant de support à la représentation religieuse qui nous intéresse ici. Or 1'arc, c'est 1 'image même, pour les Bobo, de la masculinité, car 1'arc est 1'arme des chasseurs et des guerriers, hommes aux vertus pleinement masculines dont la vocation est de parcourir le domaine de soxo -- domaine, nous le verrons, qui est aussi celui qu'occupent les génies dits "de village" : foroba wiyaxe (43. et suivants).

pô se trouve ainsi au centre d'un réseau d'interactions conceptuelles qui met en cause des catégories spirituelles qui sont profondément homologues : soxo et wiyaxe avec ses dérivés, les entités de la chasse.

pô, comme soxo et comme wiyaxe, est fortement marqué par le caractère mâle, ce qui se manifeste à la fois dans le sexe de la victime qui lui est destinée et dans le choix de sa couleur : le rouge obligatoirement.

pô, en matière de poulets exige donc des coqs rouges exclusivement et des coqs appartenant de plus à une espèce très particulière : les baba. Mais pô manifeste aussi son rapport aux entités de la chasse par le choix qu'il fait de chiens de couleur rouge en lieu et place des

30. Il y a donc plusieurs pô, chacun correspondant à l'une des 11 tribus bobo, mais tous ces pố sont d'essence unique. Pour plus de précisions sur la notion de pô et sur ses implications très diverses, $c f$. GLM 1980 : 80-86. 
caprins imposés théoriquement par le code.

Le chien joue ici le rôle d'un substitut des caprins, mais ce sont surtout ses qualités propres qui le font choisir.

Le chien, bien que domestiqué et compté comme "animal de viliage" par sa fonction de gardien, reste un "animal de brousse" par sa fonction de chasseur : c'est à ce dernier titre qu'on le verra sacrifié sur pô et bien entendu aussi sur les autres lieux de chasse (50, 53, $54,55)$.

Ainsi le caractère du chien est-i1, pour les Bobo, à double face : fidèle et obéissant, le chien est au village gardien des maisons; agressif et brutal, le chien est en brousse tueur d'animaux. C'est en tant qu'animal défenseur de l'ordre et de la paix que le chien sera sacrifié sur duba (38) par exemple ; c'est en tant qu'animal cruel et violent qu'il sera sacrifié à dwo (19-20), à certains funanyone (do, 41), mais aussi à pô, car c'est bien dans la violence qui caractérise les activités de chasse et de guerre que se forge -- sous la caution de pố-- la.solidarité tribale : le cri de ralliement lancé sur 1'autel de pô au moment du sacrifice est un cri de haine et de provocation à 1 'adresse des tribus adverses.

C'est donc, en fin de compte, sous le double signe de la chasse et de la violence que sont sacrifiés à pô des chiens mâles rouges.

Sous ce même signe de la violence s'inscrivent deux autres particularités des sacrifices faits à pô.

Première particularité : c'est sur l'autel de pô qu'on doit employer la cruelle technique d'immolation des poulets par écartèlement à vif ${ }^{31}$, technique appliquée à des coqs d'une nature hautement vénérable: les baba.

La seconde des particularités est $I^{\prime}$ absence de toute offrande faite sur l'autel de pô.

L'absence d'offrande est retenue par le code des sacrifices bobo comme I'un des facteurs entrant dans la composition des formules sacrificielles. Ce facteur "nulle offrande $(\emptyset$.$) " ne semble cependant entrete-$ nir aucun rapport évident de pertinence avec les divers autres facteurs. L'absence d'offrande coincide souvent, il est vrai, avec les facteurs de la formule 2 ( $\boldsymbol{\Delta} \mathbf{r}$ "caprins mâles rouges") et se trouve done là où

31. Cf. GLM $1981: 119$. 
1 'on attendrait 1 'offrande fogo zo (cf. non seulement pô, mais aussi foroba wiyaxe - 43 - et do - 41); cependant, des entités comme bqrè (56) et dwosa (17) qui exigent des ovins blancs refusent également toute offrande.

En fait, 1'absence d'offrande est généralement interprétée, à Kurumani notamment, comme un facteur qualitatif destiné à introduire une nuance de "dureté" dans l'image symbolique que traduit la formule sacrificielle d'une entité. Dans le cas de pô, cette "dureté" que dénoterait le refus de toute offrande coinncide parfaitement avec la violence qui caractérise l'entité et les actions qu'elle gouverne.

Tout ce que nous venons de dire de pô s'applique aux deux autres entités classées avec lui dans notre tableau 3 :

-- polo (15), en premier lieu, symbole tribal constitué par un marteau d'armes à fonction proprement rituelle dont il faut savoir qu'il entretient un rapport à dwo à l'occasion de certaines morts violentes;

-- syêsaprè (14), en second lieu, qui fait office d'autel de fondation pour la tribu et qui, de ce fait, se rattache aux saprè de lignage (57), ce qui explique que, comme eux, il demande une offrande de $f \circ g \circ$ zo.

\section{5. dwo, le mainteneur de l'ordre divin, le masque}

dwo clôt 1a liste de ces entités que nous appelons "majeures" parce que les Bobo les considèrent comme étant de même essence que wro, bien que substantiellement distinctes. dwo se présente en effet (sous l'aspect matériel du masque de feuilles) comme la théophanie ultime qui, mettant un terme aux temps cosmogoniques, consacre la naissance des temps nouveaux : ceux qu'ont vécus les honmes jusqu'à ce jour.

La figure initiale de dwo, qui se confond avec le masque de feuilles, est une entité de portée "universelle" ; elle ne possède pas d'autel fixe. Le culte de dwo s'organise donc autour de ses figures "ultérieures", c'est à dire de celles qui se sont manifestées concrètement aux hommes, après la période cosmogonique, sous la forme de masques de fibres et qui, toutes, possèdent des autels sur lesquels sont offerts des sacrifices sanglants. 
A Kurumani, deux types de figures ultérieures de dwo sont représentées : une triade dont les adeptes sont appelés sibe et un dwo de la catégorie des "dwo mâles" (dwo sini), le patama dwo.

a) La triade sibe

Les sibe, dont les membres fondateurs sont des forgerons Sanu (interdit de l'indigo), entretiennent le culte commun de trois figures de dwo révélées premièrement à Kwele (village disparu).

- kwele dwo (16)

PC - $\mathbf{A}$ b-r $\emptyset$.

kwele dwo est la première des figures de la triade sibe. Sa formule sacrificielle fait apparaître deux options antinomiques :

-- kwele dwo, bien que considéré comme mâle, demande des poules blanches -- nous sommes ainsi implicitement renvoyés à la formule 1 ;

-- Kwele dwo demande, en matière de quadrupèdes, des caprins et qui sont dûment mâles, ce qui nous renvoie cette fois à la formule 2 .

La formule de kwele dwo devrait s'écrire:

$$
\left\{\begin{array}{llll}
P & \bullet & b \\
C & \wedge & \square
\end{array} .\right.
$$

Pour simplifier, nous l'écrirons :

$$
\text { PC -A b-r } \emptyset .32
$$

En dehors du fait que des couples de facteurs opposés sont ainsi réunis dans la même formule (b / $\mathbf{r} \mathbf{A}$ ), on constate également que, contrairement à la règle, le poulet n'est pas ici en conformité de sexe et de couleur avec le quadrupède qui l'accompagne. Autre singularité, la formule sacrificielle de kwele dwo ne possède aucun facteur discriminant; on peut donc bien faire référence conjointement aux deux formules type, mais à condition de prendre celles-ci telles qu'elles se présentaient avant que ne soit introduite la notion de facteur discriminant (cf. 2.3.a).

Ces diverses singularités nous amènent à nous demander si nous ne nous trouvons pas, avec dwo, devant un cas d'espèce.

32. Rappelons que le tiret placé entre les symboles de facteurs signifie "l'un ou l'autre, selon l'espèce animale". Ici'-A signifie donc : sexe femelle pour 1 'espèce poulets, sexe mâle pour íespèce caprins ; et $b-r$ signifie blanc (poulets) et rouge (caprins). 
Notons qu'il s'agit ici d'une entité majeure, véritable centre de gravité de tout le dogme bobo. Il n'est donc pas imaginable que dwo, représentant précisément de l'ordre, gardien des équilibres préétablis, soit rejeté hors des normes. Bien au contraire, le cas de kwele dwo ne peut que refléter l'existence d'une disposition structurelle, c'est une conjoncture prévue et qui fait partie intégrante du système codifié des sacrifices.

La bipolarité de ce système, telle qu'elle est traduite par deux formules diamétralement opposées, $n$ 'en est pas pour autant remise en question. Ce que suggère plutôt la bivocité de la formule qui nous retient ici, c'est l'existence, entre les deux pôles institués, d'un secteur intermédiaire occupé par des entités qui, étant dotées d'une certaine ambivalence, peuvent conjuguer des caractères définis comme contraires. Nous aurons à y revenir, mais soulignons-le d'ores et déjà : tout ce que nous savons du système de pensée bobo, grâce à l'analyse attentive des traditions orales et des rites, tend à confirmer ces indications du code sacrificiel et à en prouver la réalité, ne serait-ce que par le rôle prééminent que jouent dans cette société les entités à vocation médiatrice.

- dwosa (17)

$$
\text { PO } \bullet \text { !b! } \varnothing \text {. }
$$

dwosa est l'une des plus prestigieuses figures de dwo; très secret, il est aussi très redouté.

Dans le choix de ses victimes, dwosa tranche avec toutes les autres figures de cwo, qu'elles soient originaires de Kwele ou d'autres lieux. La figure centrale de la triade de Kwele est seule en effet à entrer dans le cadre de la formule 1. Dans le cas de dwosa, cependant, l'accent est fortement placé sur l'appartenance féminine (sa signifie "femelle"), au point que toute victime mâle est rejetée.

L'option du facteur discriminant "couleur blanche" s'accorde parfaitement avec le sexe femelle et entraîne le choix d'un ovin. Nous retrouvons donc la formule 1 dans sa configuration naturelle (cf. 2.2.) à ceci près que, comme pour toutes les figures de dwo, les offrandes sont exclues ${ }^{33}$.

33. Tout ce qui a trait à dwo est en effet senti comme étant "dur" et grave (cf. à ce propos. 3.4.1). 


\section{- sibe dwo (18) $\quad$ PC $\bullet \mathrm{b}-\mathrm{r} \emptyset$.}

Avec sibe dwo nous retrouvons des options antinomiques présentes dans la formule sacrificielle de kwele dwo et cela bien que sibe dwo soit dans un rapport personnel beaucoup plus étroit avec dwosa : dwosa et sibe dwo émanent en effet d'un même mythe d'origine et 1 'on dit de dwosa qu'il est "la tête" de sibe dwo.

sibe dwo se rapproche donc de dwosa par son appartenance femelle très affirmée et exige, comme ce dernier, une poule blanche. En matière de quadrupèdes, c'est en revanche le sang de chèvres rouges qui seul peut couler sur 1 'autel de sibe dwo.

Le recours à des caprins nous renvoie à kwele dwo et ce sont donc, comme dans son cas, les deux formules type qui sont concernées :

$$
\left\{\begin{array}{lll}
P & \bullet \\
C & \mathrm{r}
\end{array}\right.
$$

On remarquera à ce propos qu'ici, l'entité intéressée étant femelle, la règle qui prévaut pour le caprin dans la formule 2 est appliquée et cela bien que le facteur sexuel mâle (pas plus que le facteur "couleur blanche" pour les poulets) ne soit retenu comme discriminant. On applique en somme le premier cas de figure prévu (immolation d'une chèvre rouge : cf. supra 2.3.c).

Bien que le choix d'un caprin rouge en opposition avec celui d'une poule blanche recouvre un champ de significations plus riches -auxquelles nous avons fait allusion plus haut à propos de kwele dwo -il faut noter qu'aux yeux des Bobo il fait aussi référence au fait que sibe dwo est tributaire de dwosa.

$$
\text { b) patama dwo (19-20) PC } \Delta=\mathbf{i}-\mathrm{r} \emptyset \text {. }
$$

Les dwosini, dont font partie les deux cultes du patama dwo en activité à Kurumani ( 1 'un est forob $\alpha$, c'est à dire commun à tout le village, l'autre dépend d'un unique lignage) sont, comme leur nom 1 'indique, d'appartenance mâle nettement déclarée. Les dwosîni n'en restent pas moins extrêmement indulgents sur le plan sacrificiel. En tant que mâles, ils demandent bien des caprins, mais acceptent indifféremment des victimes mâles ou femelles; il en va de même pour les poulets (avec peut-être un choix plus fréquent de femelles) dont la couleur, en outre, est totalement indifférente, dans le sens vrai du mot.

Sous les dwosini couve pourtant une grande violence (I'initiation bobo, où ils jouent un rôle central, est extrêmement rude), mais 
cette violence ne se révèle dans le sacrifice que dans des cas exceptionnels (mort par exemple d'un homme provoquée par l'une de ces figures de $(w 0)$ et se manifeste alors par l'immolation d'un chien rouge. En fait, la formule sacrificielle des dwosinni, très ouverte et ne comportant pas de facteur vraiment discriminant, rappelle, en moins absolue, celle de kwe ze dwo. Sans aller jusqu'à transgresser complètement les règles du code, patama dwo s'accomode de tout; cela tend à le situer dans cette sorte de "secteur intermédiaire" qui peu à peu se dégage, bien que déjà ici la présence de caprins et éventuellement de chiens indique un glissement vers les termes de la formule 2. La nature de dwo, nous $1^{\prime}$ avons montré ailleurs (GLM 1980 : 130 et 329), a un profond caractère de dualité : "fils" de wiro, il participe aussi, en la personne du masque notamment, de soxo. Cette particularité de dwo, qui relève du donné mythologique fondamental, fait de lui un médiateur souverain.

c) Autres éntités de la catégorie dwo (types b et c)

De nombreux autels ou objets et lieux sacrés sont en relation directe avec les diverses figures de dwo; il s'agit pour la plupart d'entités du type b (cf. 1.1.b).

Trois de ces entités relèvent de patama dwo:

- Kafuna zi (21) est le gardien du secret des connaissances relatives à dwo, c'est lui qui fait "rentrer" (dans l'oubli) tout ce qui a été divulgué. Son autel est placé sur la souche (zi) d'un arbre (kafina).

- Ko (22) est la place du village où dansent les masques, c'est aussi une entité gardienne et protectrice des porteurs de masques.

- Sifflets et tambours employés dans le culte de dwo (23 et 27). Ce sont des instruments de musique, certes, mais ils sont remarquables par le fait qu'ils sont "habités" par une entité qui, naturellement, participe de dwo.

Ces trois entités reçoivent des sacrifices dont la formule coīncide exactement avec celle de patama dwo : poule ou coq de couleur indifférente (avec préférence pour les poules dans le cas de 21). L'absence de quadrupèdes marque simplement la modeste importance de ces entités par rapport à đwo lui-même.

Deux autres entités de type b relèvent de dwosa, elles "habitent" les lieux (pati) très redoutés de l'initiation respectivement des hommes 
(sibe sine et dignitaires forkôma : 24 et 25) et des femmes (sibe dalia : 26). Le pati des sibe sîne reçoit les mêmes sacrifices que dwos $\alpha$, poules blanches et ovins, mais comme ce lieu concerne des hommes, des béliers peuvent être sacrifiés aussi bien que des brebis. Quant au pati des femmes, s'il reçoit lui aussi une poule blanche par référence à dwosa, il ne reçoit qu'une chèvre rouge eu égard à la situation sociale inférieure des femmes et à la qualité moindre de leurs connaissances religieuses.

Le cas des autels d'entités appartenant au type c (cf. 1.1.c) est plus intéressant (dwo-soxo: 28 et 29).

28 est 1 'autel du culte conjugué de dwosa et de soxo. Il reçoit une poule blanche pour le compte de dwosa, mais aussi un bouc, victime sacrificielle distinctive de soxo et à lui destinée. Poule blanche et bouc rouge coexistent à nouveau sur 1 'autel, nous renvoyant à kwe le dwo et aux commentaires que nous avions faits à son propos. Ici s'opère en effet, dans un rituel sacrificiel unique, le contact entre soxo, adepte de la formule 2, et dwosa, la figure de dwo la plus proche des entités qui, à la suite de wuro, se conforment à la formule 1. C'est là une nouvelle preuve de 1'ambivalence de dwo et de ses affinités avec soxo, mais c'est aussi et surtout, par l'existence même de ces lieux de rencontre que sont les autels dwo-soxo, une preuve de la réalité de ce "secteur intermédiaire" qui fait le pont entre les tenants de chacune des deux options sacrificielles opposées.

29 est 1 'autel du culte conjugué de patama dwo et de soxo. Les sacrifices qui y sont offerts revêtent une signification analogue à celle de 28, mais leur formule est moins parlante par le fait de 1'absence d'une victime quadrupède en raison d'un caractère localement jugé comme mineur par comparaison avec 28 . C'est une simple poule de couleur indifférente qui est donc sacrifiée ici.

Le dernier autel entrant dans la catégorie dwo est celui du devin (30). En fait, il s'agit d'un autel composite rassemblant des objets sacrés nombreux et de nature variée. Pour les Bobo, le don de voyance est le résultat d'une double influence, celle de wuro -- par 1'intermédiaire de dwo -- et celle des génies dits "de village" (foroba wiyaxe). Les ancêtres, notons-le en passant, n'inspirent pas les devins et ne sont pas particulièrement consultés par eux. La formule générale que les devins appliquent pour leurs 
sacrifices traduit surtout 1'influence de dwo puisqu'elle est conforme à celle de patama duo. Il est vrai que des sacrifices particuliers peuvent aussi être faits sur les parties de l'autel qui concernent les génies et qu'alors c'est naturellement la formule sacrificielle de ces derniers qui est retenue.

\section{6. funanyone, les "esprits"}

\section{PC $\Delta$ i, $\mathrm{n}-\mathrm{r} \varnothing .2$.}

Les funanyone sont, par rapport à wuro et à ses "fils", des entités quelque peu mineures qui se sont multipliées dans les temps postcosmogoniques et cela jusqu'à nos jours. Ce sont des "esprits" purement immatériels, des forces diffuses qui parfois se concentrent en des lieux données ou des objets et qui peuvent aussi s'emparer des êtres vivants, animaux ou hommes. Les esprits possèdent des aptitudes et des pouvoirs personnels, ils ont un caractère et des humeurs, ils peuvent même se voir reconnaître une appartenance sexuelle. On peut assez facilement entretenir des relations avec ces esprits et gagner leur confiance soit pour en obtenir des bienfaits, soit pour s'en faire des alliés auprès des entités de haut rang.

La plupart des esprits sont peu exigeants en matière de sacrifices et se contentent de poulets de $1^{\prime}$ un ou $1^{\prime}$ autre sexe, dont le plumage peut en outre être de quelque couleur que ce soit.

Quand les funanyone demandent des quadrupèdes, ce sont toujours (sauf exceptions que nous justifierons) des caprins qui leur reviennent. Les caprins conviennent aux esprits en partie parce que ces derniers sont d'importance secondaire, mais aussi et surtout parce que, étant donné leur nature et leurs fonctions, ils touchent à des domaines qui sont symboliquement associés à 1 'espèce caprine et par conséquent à la couleur rouge; d'ailleurs, quand les offrandes ne sont pas refusées, $c^{\prime}$ 'est toujours fogo $z 0$ qui est requis ${ }^{34}$ (rapport pertinent $r$ à 2 .). En dépit de ce choix des caprins, 1'appartenance mâle n'est pas dominante et ne se présente donc pas comme un facteur discriminant.

La formule sacrificielle des funanyone est pratiquement identique à celle des dwosiñi ; elle les situe dans le même secteur

34. C'est pourquoi nous plaçons les deux symboles 2. et $\emptyset$. côte à côte dans la formule sacrificielle. 
intermédiaire, avec tout de même (par l'appel qui est fait pour certains d'entre eux à des boucs rouges, voire à des chiens rouges et à du fogo zo) une tendance encore plus marquée à se rapprocher des entités concernées par la formule 2.

A Kurumani, cinq des sept funanyone recensés confirment ce que nous venons de dire :

-- Deux funanyone se contentent de simples poulets sans même une offrande :

- ôsöbeozô (31), un esprit qui donne du coeur au travail ;

- môkasa (32), un esprit qui habite un baobab et qui lance des rumeurs, divulgue des informations et des connaissances.

-- Deux autres, parmi ces cinq funanyone, reçoivent des caprins en plus des poulets et d'une offrande de fogo zo, car ils sont plus importants :

- bèro (33), un esprit guérisseur qui est aussi chasseur de sorciers ;

- kumorasiri (34), un esprit capable de conjurer la mort violente, d'apaiser les conflits.

-- Le dernier funanyonô de ce premier groupe est Lalawolo (37), sa formule sacrificielle est le simple résultat de l'application à son cas personnel de la mécanique des rapports pertinents. Lazawolo est un esprit de sexe femelle qui est exclusivement sollicité par les femmes qui ont des difficultés menstruelles. Ce sont des poules qu'il reçoit et ces poules sont noires parce que cette couleur a un rapport d'équivalence avec le rouge et que, surtout, elle est assez souvent liée à une appartenance sexuelle féminine. Le noir connote, on le sait, certaines manifestations de la féminité (les règles en particulier) qui sont, de surcroît, du domaine du secret (cf. 2.4.). C'est dans la ligne de ce même raisonnement que les victimes seront des femelles noires de l'espèce caprine, en dépit du facteur mâle discriminant, lorsque la formule 2 sera pleinement concernée (cf. 2.3.c, deuxième cas de figure : 51,52 ).

Restent deux funanyone à Kurumani dont la formule sacrificielle n'est pas conforme à la règle édictée puisque, étrangement, ils réclament des victimes blanches.

- sindo (35) est un esprit dont le culte s'est trouvé intégré 
dans le cursus initiatique.

On lui offre en sacrifice poules et béliers blancs.

- sấtogo (38) est un esprit dont le culte est, de la même façon, pris en main par les classes d'âge initiatiques ; il próside à de grandes compétitions de danse (yalza nemi dôaga).

Son autel reçoit le sang des poulets de sexe quelconque, mais de plumage toujours blanc.

La raison de cette double anomalie est simple : aussi bien sindo que sâtogo sont des esprits émanant directement de dwosa (ils sont d'ailleurs sous le contrôle du clan Sanu, indigo, "maître" de dwosa).

C'est cette extraction qui confère à d'humbles funanyone le droit de se rattacher à une formule sacrificielle aussi prestigieuse. 3.6.1. wiyaxe-funanyone

PC $\Delta=\bullet$ i $-\mathrm{r}, \mathrm{n} \emptyset .2$.

Avec les wiyaxe-funanyone, nous retrouvons des entités qui se rattachent au type $c$ de notre classification typologique et qui procèdent donc de deux catégories spirituelles à la fois, en l'occurence : des "génies" (wiyaxe) et des "esprits" (funanyone).

Esprits séjournant en brousse et partageant avec les génies divers traits de leur nature, les wiyaxe-funanyone sont les vecteurs d'une force dangereuse (le nyama) qui provoque des états de transe chez tout homme qui entre en contact avec eux. Les wiycxe-funanyone se trouvent donc au centre de ce qu'on appelle les "cultes de possession".

La formule sacrificielle de la plupart des wiyaxe-funanyone, et notamment, à Kurumani, de vyetôgo $(40)$ et do $(41,42)$, est tout à fait proche de celle des simples funanyone et ce que nous avons dit à propos de ces derniers s'applique à eux : à côté de poulets de sexe et de couleur quelconque peuvent être sacrifiés des caprins mâles et parfois des chiens (également rouges et mâles).

Le climat de violence et de frénésie dans lequel se déroulent les crises de possession s'accorde parfaitement ici avec les symboles que véhiculent les caractères spécifiques des victimes choisies : 1a couleur rouge, le sexe mâle, l'apparténance à l'espèce caprine et surtout canine, si représentative elle-même -- du moins pour une part de sa personnalité -- des comportements agressifs et brutaux.

Chez les génies wiyaxe et chez soxo, la Brousse, ces mêmes 
tendances réunies se concrétisent par l'emploi exclusif du rouge (même chez les poulets) et surtout par le choix du facteur mâle comme caractère discriminant ; cela n'est pas encore le cas pour les entités qui nous intéressent et qui restent plus proches dans leur nature des esprits que des génies et plus aptes donc à remplir le rôle qui est le leur d'intermédiaires entre les hommes et les dieux par le canal de la transe.

Seul parmi tous les wiyaxe-funanyone, le cas du très célèbre duba pose un problème, car il se présente sous un jour très particulier. duba est présent dans tous les villages bobo et son cullte est des plus anciens. duba est une entité mâle, $d u b a$ sît (38), mais il se double d'une épouse, duba ña (39).

$d u b a$ sî̀ est en rapport intime avec dwo et par là-même avec l'ensemble des entités majeures avec lesquelles il partage un penchant pour la couleur blanche et ce qu'elle recouvre au plan symbolique. duba est en effet très attentif à la pureté, à la clarté des consciences, il ne se manifeste (par des crises de possession) que si 1'ordre et l'entente règnent au village : il faut, dit-on, que personne n'ait "le coeur noir".

Tout ce qui approche duba doit être blanc, aussi bien les bandes de coton qui entourent les racines sacrées de son autel que les vêtements des possédés et des ministres de son culte. On comprend dès lors pourquoi duba exige qu'également des victimes blanches comptent parmi celles qu'on lui offre. C'est ainsi que des poulets de sexe quelconque, mais au plumage blanc immaculé, sont sacrifiés à $d u b a$, ce qui entraîne naturellement un don de sêkètè.

duba accepte néanmoins d'autres victimes et ce sont essentiellement des chiens qu'on lui réserve, des chiens qui sont ici immolés en qualité plus particulièrement de fidèles gardiens de l'ordre et de la paix. Il reste que ces chiens sont aussi retenus pour leur caractère violent et agressif puisqu'ils sont obligatoirement rouges et mâles, ce qui entraîne non moins naturellement une offrande supplémentaire, celle de fogo zo.

Que le chien soit choisi et que soient pris en compte les deux aspects quelque peu contradictoires de sa personnalité montre bien l'ambivalence profonde de duba, partagé entre les espaces clairs où règnent les forces bénéfiques d'ordre et de concorde et ceux où 
priment les forces obscures de la brousse représentées par les génies et les déchaînements de la possession.

A 1 'inverse de $d u b a$ sî̀, son épouse duba ha se place plus délibérément sur le versant qui mène aux génies wiyaxe, aux entités de la chasse et à soxo. Comme certaines femelles de ces catégories où le facteur mâle est discriminant (cf. 51 et 52), duba ho opte en effet pour l'un des choix possibles : le don de poules et de chèvres noires (cf. supra 2.3.c, deuxième cas de figure). On notera que la couleur noire, dans le cas précis de duba hâ, est symbolique non du monde de la brousse, et donc de la chasse, mais de la pluie, phénomène en relation supposée avec 1'appartenance sexuelle féminine -- de fait, duba $\hat{n} \alpha$ a le pouvoir reconnu de faire venir la pluie.

3.7. wiyaxe, les "génies"

Les génies sont les seules entités spirituelles auxquelles est prêtée une réalité tangible. Quoique difformes, ils ont en effet une apparence humaine. Les génies ont également un mode de vie très comparable à celui des hommes ; de ce fait, un réseau de relations constantes se tisse entre les deux sociétés jumelles, celle des génies et celles des hommes.

Les génies peuvent être classés selon leur lieu de résidence et surtout selon le type de rapports qu'ils ont choisi d'avoir avec les hommes : soit ils ont conservé leur autonomie totale et ils mènent une vie libre en brousse (foroba wizaxe), soit ils sont 1 iés à des groupes familiaux humains et ils résident dans les temples de lignage (wakôma wiyaxe).

\section{a) foroba wiyaxe $\mathrm{PC} ! \mathbf{\Delta !} \mathrm{r} \emptyset$.}

Ces génies hantent la brousse où ils vivent en petites communautés sur des territoires bien définis. A leur arrivée, les fondateurs d'un village doivent passer des accords avec les génies du lieu; un autel leur est alors dressé à l'extérieur du village. Ces génies sont appelés kiri tânâa kwè wiyaxe, c'est à dire "génies du village avec lesquels on s'est assis", ou encore foroba wiyaxe, c'est à dire "génies de la communauté (foroba) villageoise". Nous dirons : "génies de village".

Les foroba wiyaxe sont extrêmement vindicatifs, ils sont même 
très dangereux car imprégnés de nyama. Ces génies vivent dans une telle relation de symbiose avec la brousse (soxo) qu'ils en partagent les caractères au point que leur formule sacrificielle est rigoureusement 1 'homologue de celle: de soxo et des entités tribales du type pô. Comme ces derniers, les génies de village ont donc un caractère mâle nettement dominant et ils exigent des victimes rouges : poulets et caprins ; ils sont si "durs" que, par ailleurs, toute offrande est exclue. Ainsi, à 1 'absence près d'offrande, c'est à nouveau la formule 2 que nous retrouvons ici strictement appliquée.

A Kurumani, cette formule s'applique à tous les génies de village mâles :

- à 1'actuel kiri tânâ kwè wiyaxa (43) de Kurumani même dont le culte rassemble tous les lignages du village (foroba);

- au génie de 1'ancien village de Finiwala (46) dont I'autel a été transporté à Kurumani et dont le culte est assuré par un seul 1ignage, celui des Dao Pyêta kôma, les anciens fondateurs de Finiwala ; - à un génie (47 a) dont le lieu de résidence est une colline (tolo) d'où sort un marigot aux eaux bouillonnantes (foxoba).

A côté de ces génies mâles existent des génies femelles ${ }^{35}$ qui sont soit les épouses des génies mâles ( 47 b), soit indépendantes ( 44 , 45). Comme la formule sacrificielle de la catégorie "génies" est caractérisée par la présence du facteur mâle discriminant (F 2), le problème des femelles s'est posé et dans des termes que nous avons déjà exposés (cf. 2.3.c).

Chez les wiyaxe, le caractère sexuel est si prégnant que c'est aux troisième cas de figure que les Bobo ont fait appel, c'est à dire que doivent être sacrifiés à ces génies femelles des animaux femelles. En appliquant "mécaniquement", si 1 'on peut dire, le jeu des rapports de pertinence, la femelle entraine la couleur blanche, le blanc une victime ovine et cette dernière une offrande de sêkèzè ; paradoxalement, c'est à 1 a formule 1 que nous sommes ainsi renvoyés.

Sont dans ce cas à Kurumani, c'est-à-dire demandent poules

35. Les femmes ne peuvent s'approcher des autels des génies mâles et elles n'en consomment pas la chair sacrificielle. A l'inverse, les fenmes - et les enfants -- participent aux sacrifices sur les autels des génies fenelles. 
et brebis blanches :

- Kunuma kwê (44), génie du premier lieu de fondation de Kurumani (kunuma), quoique femelle et adepte du blanc, c'est un génie extrêmement agressif ;

- tôkatsa (45), simple génie de brousse résidant près d'un arbre où est placé son autel ;

- foxoba tolo (47 b), épouse du génie mâle du même nom (47 a), foxoba tolo est en fait le génie de la rivière bouillonnante foxoba ; c'est à ce double titre donc qu'il exige des victimes ovines blanches et du sêkèzè. Au titre du raisonnement rappelé plus haut et qui se fonde sur les conséquences d'un rapport préférentiel au sexe mâle, mais au titre aussi d'une relation naturelle à l'entité zo. Les wiyaxe aquatiques, comme foxoba tolo, sont maîtres du lit des cours d'eau, mais c'est 1 'entité majeure zo qui se confond avec l'eau elle-même (cf. note 10). La formule sacrificielle de foxoba tolo femelle satisfait bien à ce double rapport aux wiyaxe femelles et à zo. I1 est intéressant de noter en outre que si 1 'on fait un sacrifice comnun à foxoba tozo mâle et femelle, c'est un bélier que I'on sacrifie, car zo, entité majeure, l'emporte sur wiyaxe, notablement moins important.

b) wakioma wiyaxe PO $\triangle=\bullet ! \mathrm{b} ! 1$

Ces génies, qui constituent souvent des couples mâles et femelles, ont été rencontrés en brousse par le membre d'un lignage (wakôma). A la demande de ce dernier et avec l'accord de soxo, ils ont consenti à suivre 1'homme: -- ils viennent donc résider au village dans un autel que l'on érige dans le temple de lignage (wasa). Ces génies, qui sont surtout protecteurs des champs et des travaux agricoles du lignage, sont doux et bienveillants. De ces génies de 1ignage, on peut dire qu'ils sont, en quelque sorte, "passés du côté des hommes" et c'est ce qui exp1ique que, comme les ancêtres mêmes du lignage qu'ils protègent (bqrè, 56), ils reçoivent des sacrifices de poulets et d'ovins blancs accompagnés, dans leur cas, de sêkèlè.

A Kurumani, les autels des génies de lignage présents dans les divers wasa sont si nombreux qu'ils n'ont pas été recensés, mais leur formule sacrificielle est toujours conforme à la règle énoncée, c'est à dire, en fait, à la formule 1. Le sexe des poulets et des ovins correspondant, rappelons-le, au sexe du génie destinataire du sacrifice. 
Deux autels un peu particuliers ont été cependant relevés :

- wuro sa (48) est le nom donné à deux statuettes de cuivre trouvées en brousse par un ancêtre du lignage Keita Kyê ta kôma et prétendument tombées du ciel (d'où leur nom, wuro, signifiant ici le "cjel"). Pareille découverte est toujours considérée comme une manifestation matérielle de l'entité spirituelle.

La plus importante des statuettes-génies est femelle et elle reçoit, comme il se doit, des poules et des brebis blanches. La statuette mâle manifeste bien, quant à elle, qu'elle appartient à la catégorie des génies de brousse puisque, comme eux, elle réclame des coqs rouges.

- n̂año ma (49) est le nom donné à deux autres statuettes trouvées dans des conditions tout aussi fabuleuses par les Sanu kivi ta kôma. Ces statuettes représentent des veaux mâle et femelle, d'où leur nom (de nyôga nô, "petit de bovidé"). Les sacrifices qu'on leur offre sont conformes à la règle commune des génies de lignage; coq blanc pour la statuette mâle, poule et bélier blancs pour la statuette feme11e.

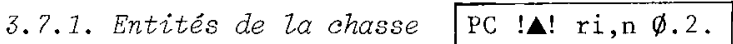

Les entités spirituelles qui gouvernent la chasse, c'est à dire qui contrôlent $1^{\prime}$ acte de mise à mort des animaux sauvages, dérivent des entités de la catégorie wiyaxe et, comme ces dernières elles-mêmes, elles ont d'étroits rapports avec les entités de la catégorie soxo, ainsi qu'avec les entités tribales qui régentent l'espace de nature où vivent librement les animaux.

Conformément à cette filiation reconnue, la formule sacrificielle des entités de chasse se rapproche de la formule de type 2. Le caractère mâle est discriminant (la chasse est d'ailleurs 1'affaire exclusivement des hommes), il entraîne donc le choix de victimes mâles et rouges qu'accompagnent le plus souvent des offrandes de fogo zo.

En matière de poulets, ce sonț des coqs qui sont impérativement recommandés (bien qu'en cas de manque absolu des poules soient acceptées pour des sacrifices courants). En matière de quadrupèdes, ce ne sont pas des caprins qui sont sacrifiés comme sur les autels des foroba wiyaxe ou de soxo, mais des chiens comme sur les autels des entités tribales. 
En dehors de sa fonction "villageoise" de gardien, le chien est, avons-nous dit, un féroce chasseur et c'est à ce titre qu'il est sacrifié aux entités de 1 a chasse (comme il 1'est, pour des raisons analogues, sur les autels de pố, par exemple). Normalement, bien entendu, ces chiens sacrifiés sont mâles et rouges.

L'un des cultes de chasseurs les plus actifs et les plus répandus aussi bien chez les Bobo que chez leurs voisins est celui de nâko (50). Le pouvoir de nâko couvre exclusivement le domaine des animaux fouisseurs, il règne sur les terriers et patronne les chasses que les Bobo affectionnent le plus : celle de l'oryctérope et celle surtout du porc-épic. Le culte de nâko est très populaire et très ouvert ; nâko, d'ailleurs, est la moins exigeante des entités de chasse puisque, seul dans son cas, il accepte indifféremment coqs et chiens de quelque couleur que ce soit.

bâkoro (52), comme nâko, possède une sorte de parèdre qui se nomme $d i b i$ (obscurité, secret). Les dibi sont très puissants et ils ont un culte très confidentiel et difficile d'accès (d'où leur nom). Le dibi de bâkoro (53) est mâle et sa compétence s'étend, comme celle de bâkoro, exclusivement aux chasses à I'arc et au fusil. dibi de bâkoro exige des coqs et des chiens rouges.

dabu (54) intervient dans 1 a chasse, mais aussi dans la guerre; son culte comporte plusieurs singularités. De fait, s'il demande des coqs et des chiens rouges lui aussi, dabu affectionne également les chats, mais de couleur noire obligatoirement. Comme les chiens, les chats sont symboliquement liés aux activités de la chasse, mais les sacrifices de chats sont très rares (c'est d'ailleurs le seul cas porté à notre connaissance). Au surplus, I'autel de dabu ne peut être touché par le sang; aussi la technique de mise à mort adoptée tant pour les coqs que les chiens et les chats est-elle celle d'une immolation "sans intervention humaine décelable" (GLM 1981 : 120).

wosîni (55) est un culte d'origine étrangère (Sénoufo-Nanergé) adopté par les Bobo. wosini propitie la chasse, mais aide aussi à la détection des sorciers.

Les entités protecrices des chasseurs sont en général de sexe mâle, c'était le cas de celles que nous venons d'énumérer. Il existe néanmoins quelques entités femelles et, comme elles appartiennent à une catégorie qui donne une valeur discriminante au sexe mâle (F 2), 
la question s'est posée du choix des victimes et surtout de leur couleur. Les formules sacrificielles des deux entités femelles dont les autels sont à Kurumani entrent dans le deuxième cas de figure prévu (cf. supra 2.3.c), elles comptent donc plusieurs des facteurs présents dans la formule 2 bis : des poules noires, des chèvres noires et du fogo an.

bôkłoro (52) suit fidèlement ces prescriptions de même que le dibi de nâko (51) qui est considéré, bien que femelle, comme plus puissant que nâko lui-même ; il intervient plus particulièrement dans le terrier même où se cache le porc-épic. Les victimes de dibi de nâko, comme celles de dabu, sont immolées sans intervention apparente.

3.8. borè, les ancêtres

PO $\Delta=\square ! \emptyset$.

Bien qu'après leur mort ils ne cessent d'être simplement des hommes, les ancêtres sont comptés par les Bobo au nombre des entités spirituelles. D'une part, l'homme a été pensé et voulu par wuro comme créature privilégiée et il a hérité d'une parcelle de divinité, d'autre part, 1'homme accède par la mort au monde invisible où règnent les entités spirituelles et il acquiert la possibilité d'entrer ainsi en contact avec $\in 1$ les. De sa nouvelle condition, le défunt tire des pouvoirs supra-naturels qui lui permettent en particulier de rester en rapport avec les hommes vivants et d'assurer la liaison entre les deux mondes.

Deux autels bien différenciés concernent les ancêtres : b̧rè (56) et saprè (57).

bqrè (de la raeine bq, "engendrer, nâ̂tre") désigne l'ensemble de ceux qui ont été engendrés dans le cadre de la parenté et à 1 'échelle soit du clan, soit du lignage. borè, ce sont donc les "ancêtres", dans le sens de totalité des ascendants défunts.

L'autel de bqrè est une simple pierre posée sur le seuil de la maison de lignage (wasa), lieu où précisément sont enterrés le fondateur du lignage et tous ses successeurs ayant reçu l'investiture sacrée (sapra).

Sur l'autel bqrè on s'adresse à l'ensemble des parents défunts, aussi bien à ceux qui ont eu la charge de diriger le lignage qu'aux plus humbles de ses membres. 
Sachant que pratiquement aucun acte, même banal, de la vie sociale, économique et surtout religieuse ne peut être décidé sans consultation des ancêtres, on imagine aisément combien fréquents sont les sacrifices faits sur b̧rè. L'accès à l'autel est d'ailleurs très facile, tout membre du lignage, homme ou femme, pouvant, pour quelque motif qui lui semble bon, être sacrifiant. Le sacrificateur est en principe le chef de lignage, mais il peut se faire remplacer par 1'un de ses nombreux adjoints.

Couramment, ce sont simplement des poulets qui sont offerts ; pour des sacrifices plus importants, des quadrupèdes sont nécessaires.

$C^{\prime}$ 'est $1 \mathrm{a}$ couleur blanche qui est requise et donc la formule 1 qui est appliquée : poulets et ovins, en principe de sexe indifférent, ce qui est vrai pour les poulets, mais non pour les ovins dont le sexe est le plus souvent mâle. Il est vrai que borè représente tout aussi bien les hommes que les femmes défunts du lignage, on ne peut donc lui attribuer un sexe plutôt que $I^{\prime}$ autre; mais les hommes ayant, dans ce type de société, le pouvoir et le prestige que l'on sait, les victimes mâles, et donc ici les béliers, ont une incontestable faveur.

Le sacrifice d'une brebis pourrait se concevoir, mais, disent les Bobo, ce serait comme "souffler sur une blessure" : cela peut apaiser, mais ne guérit aucunement. Le principe s'applique aussi à kini (cf. infra) et à beaucoup d'autres cas, notamment ceux pour lesquels nous avons employé le symbole (+) signifiant "par défaut ou en second". En d'autres termes, un anima1 non conforme aux prescriptions du code peut être parfois immolé; mais ce n'est alors qu'une solution d'attente, à charge pour l'intéressé de fournir au plus tôt la victime requise.

Le choix pour la formule sacrificielle des ancêtres du facteur discriminant "couleur blanche", avec ce qu'il recouvre au plan symbolique et au plan de la hiérarchie taxinomique, est très révélateur de l'idée que se font les Bobo de la condition humaine. Les ancêtres relèvent en effet d'une catégorie qui partage ses options sacrificielles distinctives avec des catégories qui comptent en leur sein les entités du plus haut niveau, zo par exemple, et, dans une certaine mesure, wuro lui-même. S'il n'y a pas proprement une identité de nature entre les membres de ces catégories homologues, au moins y a-t-il partage d'un certain nombre de caractères et commune appartenance à l'un des deux volets de la classification dualiste qui structure le système symbolique 
bobo. Les ancêtres sont "du côté" de wuro et de ce fait du côté également des principes qui gouvernent la vie communautaire de la société.

C'est à ce dernier titre que cette manifestation de la vie.socialisée des hommes qu'est le "village", kimi, se voit offrir sur 1'autel qui le représente (59) des béliers comme les ancêtres eux-mêmes.

kimi reçoit aussi des taureaux, c'est le seul cas attesté de la mise à mort sacrificielle de cet animal qui est peu familier aux Bobo, lesquels ne sont en effet nullement des éleveurs.

En matière d'offrandes, les deux types d'offrandes sont acceptées par kiri, non conjointement comme tokwiyanô (3), mais successivement : fogo zo pour accompagner les poulets et surtout le taureau -qui remplace en quelque sorte le caprin -- ; sêkèzè pour le bélier. saprè (57) est également un autel des ancêtres, mais ceux-ci étant considérés sous un tout autre angle. saprè vient de pro, pl. prè, qui signifie mûr, âgé ; c'est un terme qui ne désigne en fait que les hommes âgés ayant été investis religieusement du pouvoir de chef de lignage avant leur mort. L'autel des saprè ne concerne donc que ces derniers et ne peut y sacrifier (et même y faire office de sacrifiant) que le chef de lignage en exercice et lui seul.

scprè reçoit peu de sacrifices, il est réputé "dur", car "il ne donne rien" (ni la santé ni des enfants ni des biens matériels). En fait, saprè est seulement consulté dans des cas graves de rupture de l'ordre ayant été commise dans le cadre exclusivement de la parenté (crimes et transgressions diverses que saprè précisément peut sanctionner par des malheurs, des maladies ou même la mort).

scprè joue naturellement un rôle essentiel au moment de la nomination rituelle des chefs de lignage (sapra zô dâga), mais saprè est sollicité aussi à un moment très significatif : celui où se fait le sacrifice annuel à la brousse : soxo pene tyere. Le rapport des ancêtres saprè à soxo est reconnu et c'est lui qui justifie les caprins mâles et rouges et le fogo zo qui sont offerts sacrificiellement sur I'autel saprè.

A nouveau nous trouvons ici une ambivalence manifeste : sous 1 'angle de la génération les ancêtres se réclament de la formule 1 , sous l'angle du pouvoir ils en appellent à la formule 2 .

Que le pouvoir soit symboliquement du côté du rouge, des caprins et du fogo zo, et donc du côté de la violence, de la virilité, de la 


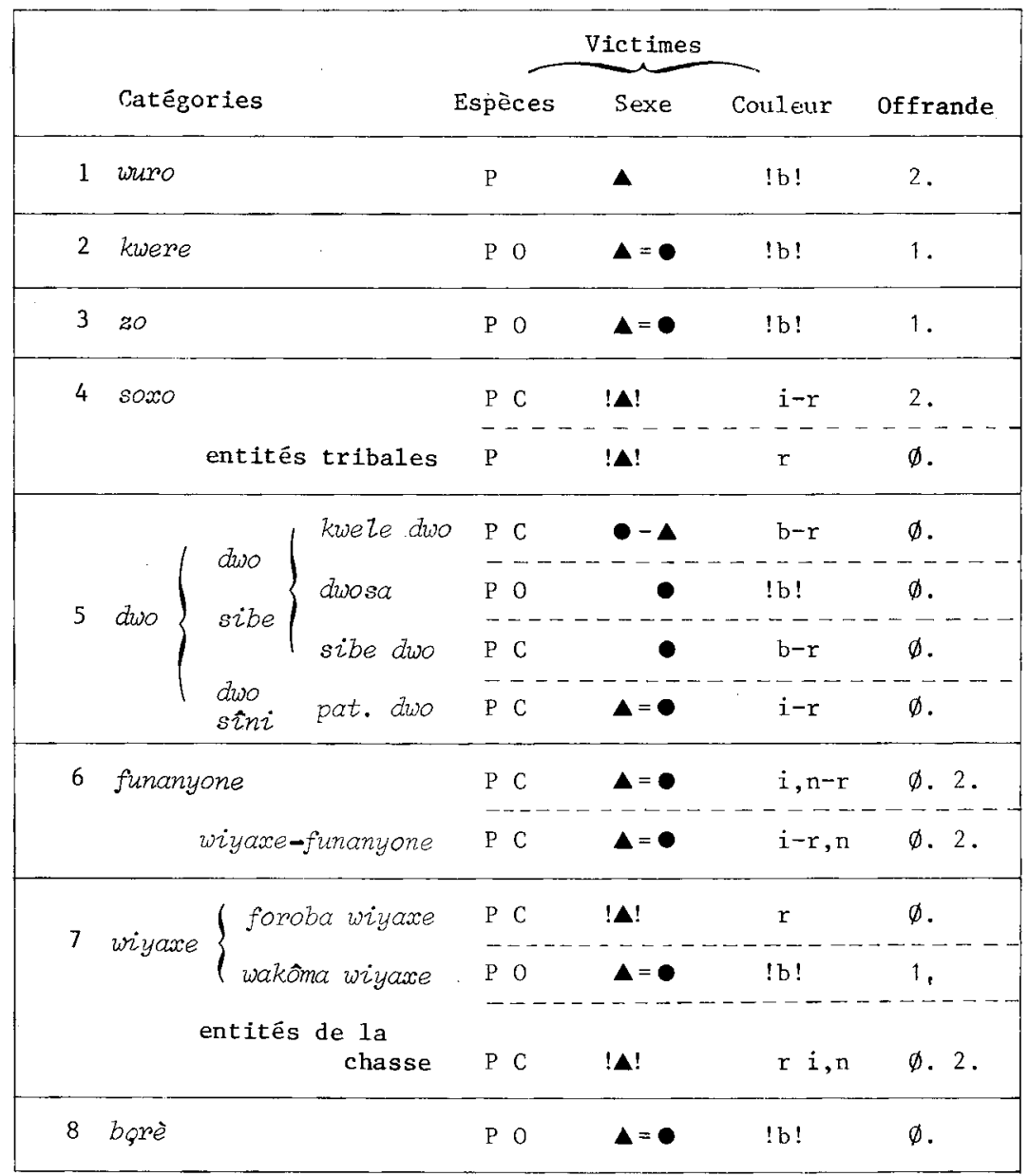

\begin{tabular}{|c|c|}
\hline $\mathbf{\Delta}=\mathbf{0}$ & $1^{\prime}$ un ou $1^{\prime}$ autre, indifféremment \\
\hline$\Delta-0 \quad b-r$ & $I^{\prime}$ un ou $I^{\prime}$ autre, selon 1 'espèce \\
\hline$\Delta$ & mâle exclusivement \\
\hline$\bullet$ & femelle exclusivement \\
\hline, $\mathrm{n}$ & dans le cas d'une entité femelle \\
\hline ! ! & facteur discriminant \\
\hline
\end{tabular}


dureté, n'étonne pas quand on sait quelle attitude critique et quelle méfiance profonde les Bobo ont pour toutes formes d'autorité.

Subsidiairement, les caprins marquent aussi la subordination de saprè à bqrè : le lien charnel de postérité prime l'exercice concédé de 1'autorité, en d'autres termes, l'existence et la perpétuation de 1 a lignée sont de plus d'importance que le pouvoir temporel exexcé un temps sur ses membres.

La terrasse $(z \hat{O})$ où se réunissent pour délibérer les chefs des lignages composant le village est un lieu sacré qui s'appelle sapra zô (58) ; il est en rapport direct avec la pratique de la chefferie et donc avec saprè, c'est pourquoi les poulets qu'on y sacrifie sont rouges.

Au nombre des entités du type "d", il y a kimi dont nous avons déjà parlé et qui dérive directement de boré (comme d'ailleurs kiri kôndigö qui est le lieu de réunion des arbitres judiciaires du village); mais il y a aussi -- dernière des entités que nous avons recensées à Kurumani -- les jumeaux : kiuiye (61).

Ces êtres remarquables sont évidemment une image idéale de 1a génération, le modèle de toute procréation et donc une parfaite représentation des bqrè ; c'est à ce titre que $1^{\prime}$ autel des jumeaux reçoit des poulets blancs -- nés de la même couvée et en quelque sorte donc jumeaux. L'aîné des jumeaux est toujours appelé $s \hat{\imath} \hat{\imath}$ ("mâle", quel que soit son sexe réel) et c'est un coq qui lui est sacrifié. Le cadet est appelé zo, il est considéré comme de sexe femelle et reçoit une poule.

Un bouc est aussi sacrifié sur $1^{\prime}$ autel des $s \hat{\imath} \hat{\imath}$, avec accompagnement d'une offrande fogo zo. La raison de ce choix n'est pas très claire, le rapport à saprè est possible, mais il semble plutôt que ce soit, par l'intermédiaire de ce dernier, une relation à soxo qui s'exprime ou, plus exactement, une relation aux foroba wiyaxe (qui sont, on le sait, eux-mêmes en rapport avec soxo). Cette hypothèse est confirmée par le fait que beaucoup de jumeaux sont considérés comme étant des génies incarnés.

\section{L'IVTERPRETATION}

La mise en application du code sacrificiel et des principes théoriques qu'il traduit nous a apporté -- à travers 61 exemples successifs -- un ensemble de formules symboliques qui demandent à être 
analysées et ordonnées dans la perspective d'une interprétation générale.

Parmi les plus significatives des données que le décryptage de ces formules sacrificielles propose à l'analyse, il y a la confirmation de ce que nous savions de longue date par d'autres voies que le sacrifice (par les mythes notament) : 1'existence de deux univers aux tendances contrastées. Ce qu'en revanche nous connaissons mieux désormais, c'est la liste précise des tenants de ces deux univers et les traits spécifiques de leur caractère, tels qu'ils apparaissent à la.. lecture des symboles véhiculés par les facteurs de leurs formules sacrificielles.

Ce que, dans son application, le code sacrificiel bobo nous révèle aussi à ce même propos, c'est l'existence bien établie de ce que nous avons appelé "un secteur intermédiaire", d'un domaine neutre participant également des deux univers antagonistes et où des forces d'équilibre oeuvrent sous 1 'espèce d'entités à vocation médiatrice.

L'image de cette structure dualiste "tempérée" restait, il faut le reconnaître, un peu floue tant que $1^{\prime}$ 'on s'en tenait au classement des entités spirituelles qui nous a servi de guide jusqu'ici (cf. supra 1.1.a) 'et que 1 'on retrouve, accompagné des formules sacrificielles correspondantes, dans le tableau récapitulatif 4 .

Ce classement, sur lequel nous nous sommes fondé au départ de nos enquêtes sur le sacrifice, était issu, lui aussi, des enseignements fournis principalement par les mythes cosmogoniques bobo. Il.s'agissait surtout, en l'espèce, d'un tableau de la chronologie des manifestations premières des principales entités spirituelles: Ce tableau, qui nous avait notamment permis de dessiner le circuit de la prière et des actes sacrificiels et de dresser un organigramme des cultes (GLM 1980 : 157 et 159), garde d'ailleurs toute sa valeur en tant que représentation * dynamique des relations mutuelles entre les hommes et les diverses catégories spirituelles.

C'est une image beaucoup plus nette de la structure même du système d'ensemble des croyances bobo qui nous est offerte aujourd'hui au terme de notre recherche sur les modalités du sacrifice. Le tableau 5 en témoigne.

Pour réaliser ce nouveau tableau, il nous a suffi, en partant des deux formules sacrificielles types $F 1$ et $F 2$, de répartir en deux 


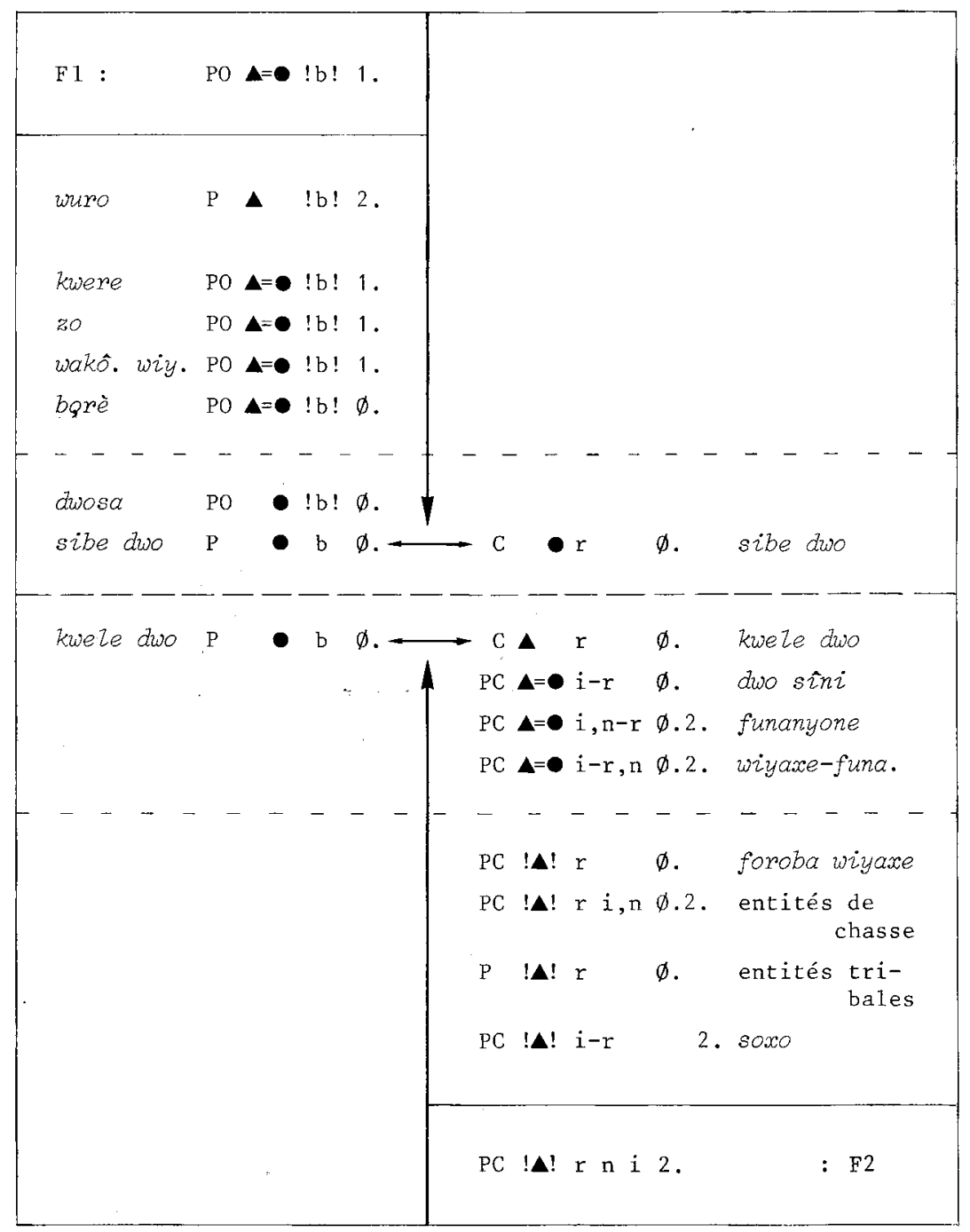


séries, selon leur conformité respective, les formules personnelles de chacune des principales entités spirituelles, puis de les distribuer dans l'ordre établi sur deux vecteurs de sens opposé.

Le premier vecteur, partant de la formule 1 , passe d'abord par wuro -- que sa qualité de divinité suprême place naturellement en têté, en dépit d'une formule sacrificielle quelque peu discordante -- puis rencontre successivement les quatre grandes entités également conformes aux termes de la formule 1 : kwere, zo, wakôma wiyaxe et bqrè.

Le second vecteur, partant de la formule 2, passe d'abord par soxo, qui fait appel à une formule homologue terme à terme, puis par les entités tribales, celles de la chasse et enfin par les foroba wiyaxe, tous utilisateurs de formules sacrificielles proches du modèle F 2 .

Avant même que les deux vecteurs ne se rencontrent, ils butent sur des formules qui ne peuvent s'écrire que transversalement, concrétisant ainsi l'existence de cette zone charnière que nous avons appelée "secteur intermédiaire".

Si dwosa en effet est encore placé sur le vecteur de F 1, sibe dwo et kwele dwo ne le sont plus que pour moitié. Du côté des poulets sacrificiels, la couleur blanche est bien requise, mais du côté des quadrupèdes, ce sont les caprins et la couleur rouge distinctifs de F 2 qui sont présents. Aux approches du point de rencontre des vecteurs, F 2 lui-même a perdu la rigueur de certaines de ses options comme le caractère discriminant de l'appartenance mâle de ses victimes sacrificielles.

Que 1'on remplace le nom des entités par les concepts qu'elles représentent, que 1 'on substitue aux termes des formules les symboles et le sens qu'ils portent, et c'est toute une axiomatique qui se dégage de cette construction de l'esprit, éclairant de ses propositions incitatives le sens des discours et des conduites.

La place ici nous fait défaut pour entrer plus avant dans I'exploitation du vaste donné ainsi acquis par 1'analyse de pratiques sacrificielles banales et quotidiennes pourtant. Dans un prochain article nous reprendrons ce sujet et chercherons à définir, en conclusion à cette enquête, ce que recouvre, pour les Bobo, 1'acte de sacrifier.

(A suivre) 


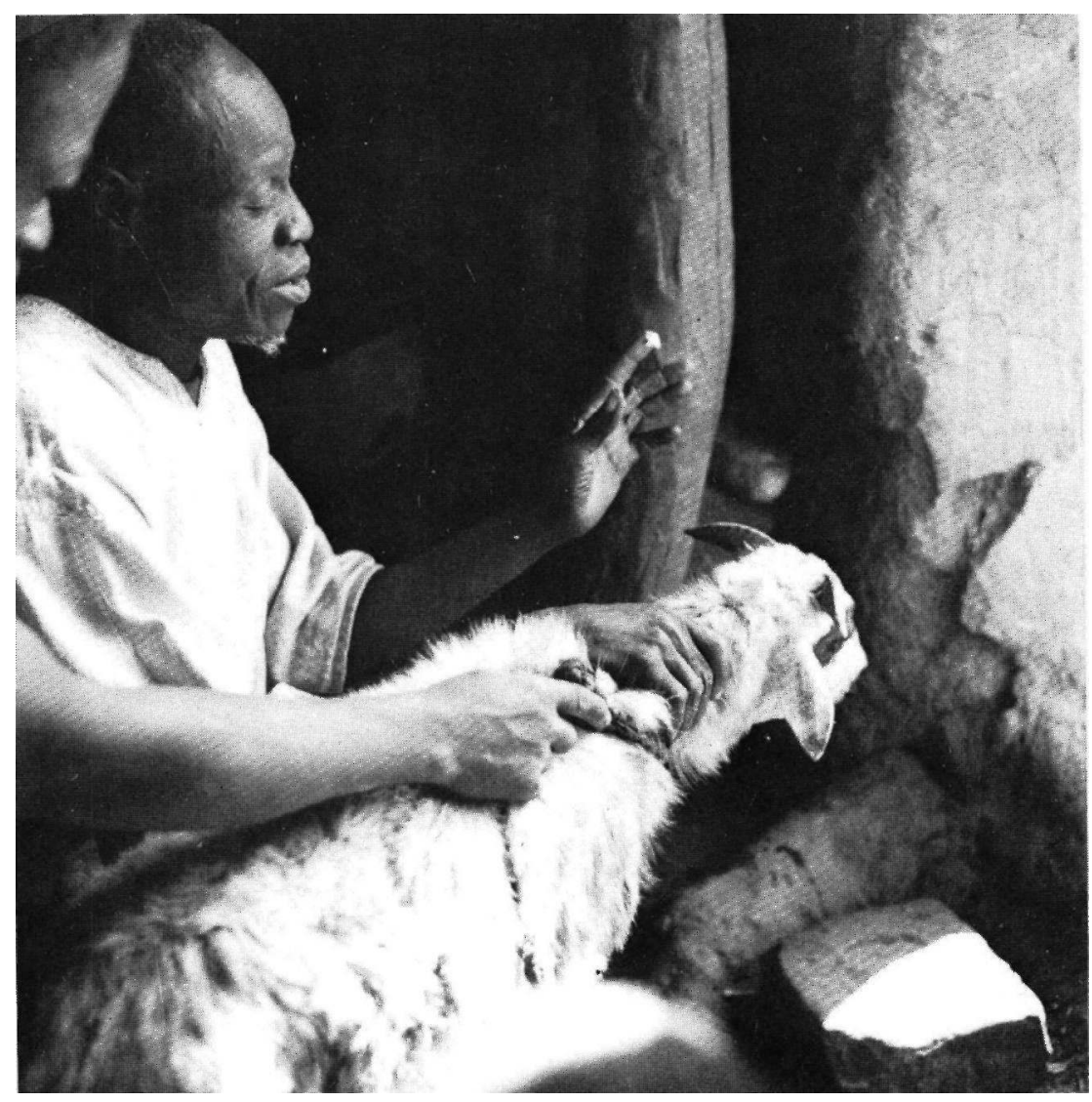

Photo 1. Présentation du bélier blanc devant bqvè, l'autel des ancêtres (pierre du seuil). 


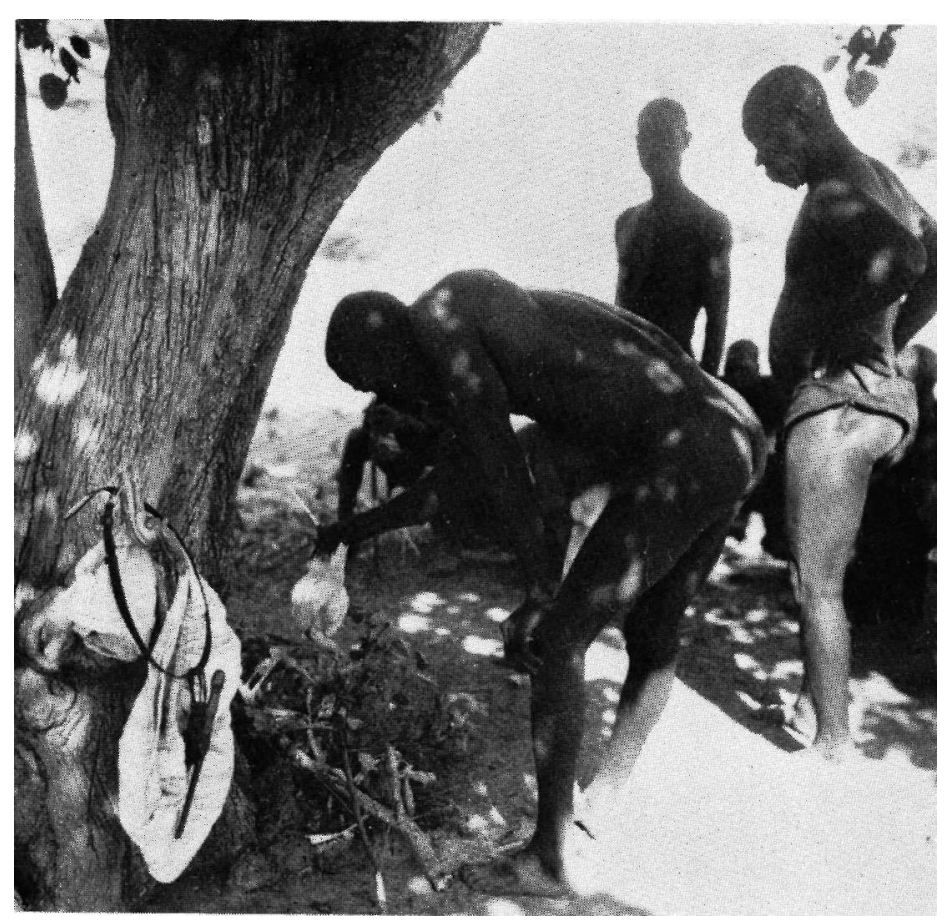

Photo 2. Sacrifice d'un poulet bicolore (rouge et blanc) sur l'autel villageois de la brousse (kiri tânâ kwè soxo) .

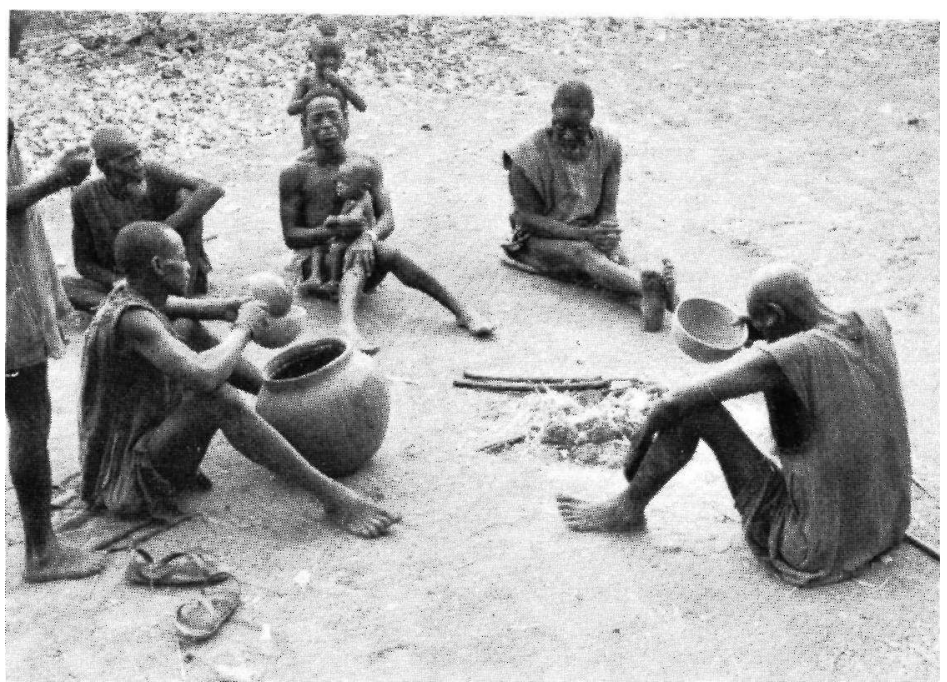

Photo 3. Consommation de bière de mil à la fin d'un sacrifice sur l'autel du patama dwo (noter la présence des deux flûtes sacrées de dwo sur l'autel). 